\title{
Flag Partial Differential Equations and Representations of Lie Algebras
}

\author{
Xiaoping $\mathrm{Xu}^{2}$ \\ Institute of mathematics, Academy of Mathematics \& System Sciences \\ Chinese Academy of Sciences, Beijing 100080, P. R. China
}

\begin{abstract}
In this paper, we solve the initial value problems of variable-coefficient generalized wave equations associated with trees and a large family of linear constant-coefficient partial differential equation by algebraic methods. Moreover, we find all the polynomial solutions for a 3-dimensional variable-coefficient flag partial differential equation of any order, the linear wave equation with dissipation and the generalized anisymmetrical Laplace equation. Furthermore, the polynomial-trigonometric solutions of a generalized Klein-Gordan equation associated with 3-dimensional generalized Tricomi operator $\partial_{x}^{2}+x \partial_{y}^{2}+y \partial_{z}^{2}$ are also given. As applications to representations of Lie algebras, we find certain irreducible polynomial representations of the Lie algebras $\operatorname{sl}(n, \mathbb{F}), \operatorname{so}(n, \mathbb{F})$ and the simple Lie algebra of type $G_{2}$.
\end{abstract}

\section{Introduction}

Barros-Neto and Gel'fand [BG1,BG2] $(1998,2002)$ studied solutions of the equation

$$
u_{x x}+x u_{y y}=\delta\left(x-x_{0}, y-y_{0}\right)
$$

related to the Tricomi operator $\partial_{x}^{2}+x \partial_{y}^{2}$. A natural generalization of the Tricomi operator is $\partial_{x_{1}}^{2}+x_{1} \partial_{x_{2}}^{2}+\cdots+x_{n-1} \partial_{x_{n}}^{2}$. The equation

$$
u_{t}=u_{x_{1} x_{1}}+u_{x_{2} x_{2}}+\cdots+u_{x_{n} x_{n}}
$$

\footnotetext{
${ }^{1} 2000$ Mathematical Subject Classification. Primary 17B30, 35F15, 35G15; Secondary 35C15, 35Q58

${ }^{2}$ Research Supported by China NSF 10431040
} 
is the well known classical heat conduction equation related to the Laplacian operator $\partial_{x_{1}}^{2}+\partial_{x_{2}}^{2}+\cdots+\partial_{x_{n}}^{2}$. As pointed out in [BG1, BG2], the Tricomi operator is an analogue of the Laplacian operator. In [X2], we have solved the following natural analogue of heat conduction equation:

$$
u_{t}=u_{x_{1} x_{1}}+x_{1} u_{x_{2} x_{2}}+\cdots+x_{n-1} u_{x_{n} x_{n}} .
$$

Indeed we have solved analogous heat conduction equations related to more general generalized Tricomi operators associated with tree graphs.

A tree $\mathcal{T}$ consists of a finite set of nodes $\mathcal{N}=\left\{\iota_{1}, \iota_{2}, \ldots, \iota_{n}\right\}$ and a set of edges

$$
\mathcal{E} \subset\left\{\left(\iota_{i}, \iota_{j}\right) \mid 1 \leq i<j \leq n\right\}
$$

such that for each node $\iota_{i} \in \mathcal{N}$, there exists a unique sequence $\left\{\iota_{i_{1}}, \iota_{i_{2}}, \ldots, \iota_{i_{r}}\right\}$ of nodes with $1=i_{1}<i_{2}<\cdots<i_{r-1}<i_{r}=i$ for which

$$
\left(\iota_{i_{1}}, \iota_{i_{2}}\right),\left(\iota_{i_{2}}, \iota_{i_{3}}\right), \ldots,\left(\iota_{i_{r-2}}, \iota_{i_{r-1}}\right),\left(\iota_{i_{r-1}}, \iota_{i_{r}}\right) \in \mathcal{E}
$$

We also denote the tree $\mathcal{T}=(\mathcal{N}, \mathcal{E})$. For a tree $\mathcal{T}=(\mathcal{N}, \mathcal{E})$, we call the differential operator

$$
d_{\mathcal{T}}=\partial_{x_{1}}^{2}+\sum_{\left(\iota_{i}, \iota_{j}\right) \in \mathcal{E}} x_{i} \partial_{x_{j}}^{2}
$$

a generalized Tricomi operator of type $\mathcal{T}$. In [X2], we have solved the following analogue of heat conduction equation:

$$
u_{t}=d_{\mathcal{T}}(u)
$$

subject to the initial condition:

$$
u\left(0, x_{1}, \ldots, x_{n}\right)=f\left(x_{1}, \ldots, x_{n}\right) \quad \text { for } x_{i} \in\left[-a_{i}, a_{i}\right]
$$

where $f$ is a given continuous function and $a_{i}$ are given positive real constants.

The equation

$$
u_{t t}-u_{x_{1} x_{1}}-u_{x_{2} x_{2}}-\cdots-u_{x_{n} x_{n}}=0
$$

is the well-known wave equation associated with the Laplace operator $\partial_{x_{1}}^{2}+\partial_{x_{2}}^{2}+\cdots+\partial_{x_{n}}^{2}$. One of our goals in this paper is to solve the generalized wave equation

$$
u_{t t}-d_{\mathcal{T}}(u)=0
$$

subject to the initial conditions:

$$
u\left(0, x_{1}, \ldots, x_{n}\right)=g_{0}\left(x_{1}, \ldots, x_{n}\right), \quad u_{t}\left(0, x_{1}, \ldots, x_{n}\right)=g_{1}\left(x_{1}, \ldots, x_{n}\right)
$$

for $x_{i} \in\left[-a_{i}, a_{i}\right]$, where $g_{1}, g_{2}$ are given continuous functions and $a_{i}$ are given positive real constants. This is done by using our results in [X2] and an algebraic method of solving the following differential equation of flag type:

$$
\left(d_{1}+f_{1} d_{2}+f_{2} d_{3}+\cdots+f_{n-1} d_{n}\right)(u)=0,
$$


where $d_{1}, d_{2}, \ldots, d_{n}$ are certain commuting locally nilpotent differential operators on the polynomial algebra $\mathbb{R}\left[x_{1}, x_{2}, \ldots, x_{n}\right]$ and $f_{1}, \ldots, f_{n-1}$ are polynomials satisfying

$$
d_{i}\left(f_{j}\right)=0 \quad \text { if } i>j
$$

Another slightly adjusted method enables us to solve a large family of linear constantcoefficient partial differential equations subject to initial conditions easily. This family contains almost all well-known linear constant-coefficient partial differential equations such as the Helmholtz equation and the Klein-Gordon equation. A general equation in this family can not be solved by separation of variables.

Polynomial solutions of linear partial differential equations are important for many reasons. For instance, polynomial solutions of Laplace equations are called harmonic polynomials, which are fundamental objects in differential geometry and analysis. In this paper, we have used our algebraic methods to find a basis of the space of all polynomial solutions for the following differential equations: (1)

$$
\partial_{x}^{m_{1}}(u)+x^{n_{1}} \partial_{y}^{m_{2}}(u)+y^{n_{2}} \partial_{z}^{m_{3}}(u)=0
$$

where $m_{1}, m_{2}, m_{3}$ are positive integers and $n_{1}, n_{2}$ are nonnegative integers; (2) the linear wave equation with dissipation:

$$
u_{t t}+u_{t}-u_{x_{1} x_{1}}-u_{x_{2} x_{2}}-\cdots-u_{x_{n} x_{n}}=0
$$

$$
u_{t t}+\frac{\lambda}{t} u_{t}-\epsilon\left(u_{x_{1} x_{1}}+u_{x_{2} x_{2}}+\cdots+u_{x_{n} x_{n}}\right)=0
$$

where $\epsilon \in\{1,-1\}$ and $\lambda$ is a nonzero real constant. When $m_{1}=m_{2}=m_{3}=2$ and $n_{1}=n_{2}=0,(1.14)$ is exactly the 3 -dimensional Laplace equation. If $\epsilon=-1,(1.16)$ is the generalized anisymmetrical Laplace equation (cf. [A]). Moreover, the special EulerPoisson-Darboux equation:

$$
u_{t t}-u_{x_{1} x_{1}}-u_{x_{2} x_{2}}-\cdots-u_{x_{n} x_{n}}-\frac{m(m+1)}{t^{2}} u=0
$$

can be changed into a equation of type (1.16) with $\epsilon=1$. Furthermore, we have found the polynomial-trigonometric solutions of the generalized Klein-Gordan equation

$$
u_{t t}-u_{x x}-x u_{y y}-y u_{z z}+a^{2} u=0
$$

associated with 3-dimensional generalized Tricomi operator $\partial_{x}^{2}+x \partial_{y}^{2}+y \partial_{z}^{2}$.

Flag partial differential equations also naturally appear in the representation theory of Lie algebras. Let $\mathcal{G}$ be a finite-dimensional simple Lie algebra with the Cartan root-space decomposition:

$$
\mathcal{G}=\mathcal{H} \oplus \bigoplus_{\alpha \in \Phi} \mathcal{G}_{\alpha}
$$


Suppose that $M$ is a finite-dimensional $\mathcal{G}$-module. Take a weight-vector basis $\left\{v_{1}, \ldots, v_{n}\right\}$ of $M$. Write

$$
\xi\left(v_{i}\right)=\sum_{j=1}^{n} \rho_{i, j}(\xi) v_{j} \quad \text { for } \xi \in \mathcal{G} .
$$

We define an action of $\mathcal{G}$ on $\mathbb{R}\left[x_{1}, \ldots, x_{n}\right]$ by

$$
\xi(g)=\sum_{i, j=1}^{n} \rho_{i, j}(\xi) x_{j} \partial_{x_{i}}(g) \quad \text { for } \xi \in \mathcal{G}, g \in \mathbb{R}\left[x_{1}, \ldots, x_{n}\right] .
$$

Then $\mathbb{R}\left[x_{1}, \ldots, x_{n}\right]$ forms a $\mathcal{G}$-module isomorphic to the symmetric tensor $S(M)$ over $M$. Take a base $\Pi$ of the root system $\Phi$ and $0 \neq \xi_{\alpha} \in \mathcal{G}_{\alpha}$ for $\alpha \in \Pi$. According to Weyl's theorem of completely reducibility, $\mathbb{R}\left[x_{1}, \ldots, x_{n}\right]$ can be decomposed as a direct sum of irreducible $\mathcal{G}$-submodules. Such a decomposition is completely determined by the polynomial solutions (singular vectors or highest-weight vectors) of the following system of flag partial differential equations:

$$
\sum_{i, j=1}^{n} \rho_{i, j}\left(\xi_{\alpha}\right) x_{j} \partial_{x_{i}}(u)=0, \quad \alpha \in \Pi
$$

As simple applications of our algebraic methods of solving differential equations, we find a basis for certain irreducible polynomial representations of the Lie algebras $\operatorname{sl}(n, \mathbb{F}), \operatorname{so}(n, \mathbb{F})$ and the simple Lie algebra of type $G_{2}$.

In Section 2, we will present our algebraic methods and find all polynomial solutions of the above concerned partial differential equations. Section 3 is devoted to solve two initial value problems we mentioned in the above. In Section 4, certain polynomial representations of Lie algebras will be given.

\section{Polynomial Solutions}

Take any subfield $\mathbb{F}$ of the field $\mathbb{C}$ of complex numbers. We assume all the vector spaces are over $\mathbb{F}$ unless it is specified. For a positive integer $n$, we denote by

$$
\mathcal{A}=\mathbb{F}\left[x_{1}, \ldots, x_{n}\right]
$$

the algebra of polynomials in $n$ variables. The following algebraic result is one of our key lemmas of solving concerned differential equations.

Lemma 2.1. Suppose that $\mathcal{A}$ is free module over a subalgebra $\mathcal{B}$ generated by a filtrated subspace $V=\bigcup_{r=0}^{\infty} V_{r}$ (i.e., $\left.V_{r} \subset V_{r+1}\right)$. Let $T_{1}$ be a linear operator on $\mathcal{A}$ with a right inverse $T_{1}^{-}$such that

$$
T_{1}(\mathcal{B}), T_{1}^{-}(\mathcal{B}) \subset \mathcal{B}, \quad T_{1}\left(\eta_{1} \eta_{2}\right)=T_{1}\left(\eta_{1}\right) \eta_{2}, \quad T_{1}^{-}\left(\eta_{1} \eta_{2}\right)=T_{1}^{-}\left(\eta_{1}\right) \eta_{2}
$$

for $\eta_{1} \in \mathcal{B}, \eta_{2} \in V$, and let $T_{2}$ be a linear operator on $\mathcal{A}$ such that

$$
T_{2}\left(V_{r+1}\right) \subset \mathcal{B} V_{r}, \quad T_{2}(f \zeta)=f T_{2}(\zeta) \quad \text { for } 0 \leq r \in \mathbb{Z}, \quad f \in \mathcal{B}, \zeta \in \mathcal{A} .
$$


Then we have

$$
\begin{aligned}
& \left\{g \in \mathcal{A} \mid\left(T_{1}+T_{2}\right)(g)=0\right\} \\
= & \operatorname{Span}\left\{\sum_{i=0}^{\infty}\left(-T_{1}^{-} T_{2}\right)^{i}(h g) \mid g \in V, h \in \mathcal{B} ; T_{1}(h)=0\right\},
\end{aligned}
$$

where the summation is finite under our assumption. Moreover, the operator $\sum_{i=0}^{\infty}\left(-T_{1}^{-} T_{2}\right)^{i} T_{1}^{-}$ is a right inverse of $T_{1}+T_{2}$.

Proof. For $h \in \mathcal{B}$ such that $T_{1}(h)=0$ and $g \in V$, we have

$$
\begin{aligned}
& \left(T_{1}+T_{2}\right)\left(\sum_{i=0}^{\infty}\left(-T_{1}^{-} T_{2}\right)^{i}(h g)\right) \\
= & T_{1}(h g)-\sum_{i=1}^{\infty} T_{1}\left[T_{1}^{-} T_{2}\left(-T_{1}^{-} T_{2}\right)^{i-1}(h g)\right]+\sum_{i=0}^{\infty} T_{2}\left[\left(-T_{1}^{-}\right)^{i}(h g)\right] \\
= & T_{1}(h) g-\sum_{i=1}^{\infty}\left(T_{1} T_{1}^{-}\right) T_{2}\left(-T_{1}^{-} T_{2}\right)^{i-1}(h g)+\sum_{i=0}^{\infty} T_{2}\left(-T_{1}^{-} T_{2}\right)^{i}(h g) \\
= & -\sum_{i=1}^{\infty} T_{2}\left(-T_{1}^{-} T_{2}\right)^{i-1}(h g)+\sum_{i=0}^{\infty} T_{2}\left(-T_{1}^{-} T_{2}\right)^{i}(h g)=0
\end{aligned}
$$

by (2.2). Denote by $\mathbb{N}$ the set of nonnegative integers. Set $V_{-1}=\{0\}$. For $k \in \mathbb{N}$, we take $\left\{\psi_{i} \mid i \in I_{k}\right\} \subset V_{k}$ such that

$$
\left\{\psi_{i}+V_{k-1} \mid i \in I_{k}\right\} \text { forms a basis of } V_{k} / V_{k-1},
$$

where $I_{k}$ is an index set. Let

$$
\mathcal{A}^{(m)}=\mathcal{B} V_{m}=\sum_{s=0}^{m} \sum_{i \in I_{s}} \mathcal{B} \psi_{i} .
$$

Obviously,

$$
T_{1}\left(\mathcal{A}^{(m)}\right), T_{1}^{-}\left(\mathcal{A}^{(m)}\right), T_{2}\left(\mathcal{A}^{(m+1)}\right) \subset \mathcal{A}^{(m)} \quad \text { for } m \in \mathbb{N}
$$

by (2.2) and (2.3), and

$$
\mathcal{A}=\bigcup_{m=0}^{\infty} \mathcal{A}^{(m)}
$$

Suppose $\phi \in \mathcal{A}^{(m)}$ such that $\left(T_{1}+T_{2}\right)(\phi)=0$. If $m=0$, then

$$
\phi=\sum_{i \in I_{0}} h_{i} \psi_{i}, \quad h_{i} \in \mathcal{B} .
$$

Now

$$
0=\left(T_{1}+T_{2}\right)(\phi)=\sum_{i \in I_{0}} T_{1}\left(h_{i}\right) \psi_{i}+\sum_{i \in I_{0}} h_{i} T_{2}\left(\psi_{i}\right)=\sum_{i \in I_{0}} T_{1}\left(h_{i}\right) \psi_{i},
$$

Since $T_{1}\left(h_{i}\right) \in \mathcal{B}$ by $(2.2)$ and $\mathcal{A}$ is a free $\mathcal{B}$-module generated by $V$, we have $T_{1}\left(h_{i}\right)=0$ for $i \in I_{0}$. Denote by $\mathcal{S}$ the right hand side of the equation (2.4). Then

$$
\phi=\sum_{i \in I_{0}} \sum_{m=0}^{\infty}\left(-T_{1}^{-} T_{2}\right)^{m}\left(h_{i} \psi_{i}\right) \in \mathcal{S} .
$$


Suppose $m>0$. We write

$$
\phi=\sum_{i \in I_{m}} h_{i} \psi_{i}+\phi^{\prime}, \quad h_{i} \in \mathcal{B}, \phi^{\prime} \in \mathcal{A}^{(m-1)} .
$$

Then

$$
0=\left(T_{1}+T_{2}\right)(\phi)=\sum_{i \in I_{m}} T_{1}\left(h_{i}\right) \psi_{i}+T_{1}\left(\phi^{\prime}\right)+T_{2}(\phi) .
$$

Since $T_{1}\left(\phi^{\prime}\right)+T_{2}(\phi) \in \mathcal{A}^{(m-1)}$, we have $T_{1}\left(h_{i}\right)=0$ for $i \in I_{m}$. Now

$$
\phi-\sum_{i \in I_{m}} \sum_{k=0}^{\infty}\left(-T_{1}^{-} T_{2}\right)^{k}\left(h \psi_{i}\right)=\phi^{\prime}-\sum_{i \in I_{m}} \sum_{k=1}^{\infty}\left(-T_{1}^{-} T_{2}\right)^{k}\left(h_{i} \psi_{i}\right) \in \mathcal{A}^{(m-1)}
$$

and (2.5) implies

$$
\left(T_{1}+T_{2}\right)\left(\phi-\sum_{i \in I_{m}} \sum_{k=0}^{\infty}\left(-T_{1}^{-} T_{2}\right)^{k}\left(h_{i} \psi_{i}\right)\right)=0
$$

By induction on $m$,

$$
\phi-\sum_{i \in I_{m}} \sum_{k=0}^{\infty}\left(-T_{1}^{-} T_{2}\right)^{k}\left(h_{i} \psi_{i}\right) \in \mathcal{S}
$$

Therefore, $\phi \in \mathcal{S}$.

For any $f \in \mathcal{A}$, we have:

$$
\begin{aligned}
& \left(T_{1}+T_{2}\right)\left(\sum_{i=0}^{\infty}\left(-T_{1}^{-} T_{2}\right)^{i} T_{1}^{-}\right)(f) \\
= & f-\sum_{i=1}^{\infty} T_{2}\left(-T_{1}^{-} T_{2}\right)^{i-1} T_{1}^{-}(f)+\sum_{i=0}^{\infty} T_{2}\left(-T_{1}^{-} T_{2}\right)^{i} T_{1}^{-}(f)=f .
\end{aligned}
$$

Thus the operator $\sum_{i=0}^{\infty}\left(-T_{1}^{-} T_{2}\right)^{i} T_{1}^{-}$is a right inverse of $T_{1}+T_{2}$.

We remark that the above operator $T_{1}$ and $T_{2}$ may not commute.

For convenience, we take the following notation of indices:

$$
\overline{i, j}=\{i, i+1, \ldots, j\}
$$

where $i \leq j$ are integers. Define

$$
x^{\alpha}=x_{1}^{\alpha_{1}} x_{2}^{\alpha_{2}} \cdots x_{n}^{\alpha_{n}} \quad \text { for } \alpha=\left(\alpha_{1}, \ldots, \alpha_{n}\right) \in \mathbb{N}^{n} \text {. }
$$

Moreover, we denote

$$
\epsilon_{i}=(0, \ldots, 0, \stackrel{i}{1}, 0, \ldots, 0) \in \mathbb{N}^{n} .
$$

For each $i \in \overline{1, n}$, we define the linear operator $\int_{\left(x_{i}\right)}$ on $\mathcal{A}$ by:

$$
\int_{\left(x_{i}\right)}\left(x^{\alpha}\right)=\frac{x^{\alpha+\epsilon_{i}}}{\alpha_{i}+1} \quad \text { for } \quad \alpha \in \mathbb{N}^{n}
$$


Furthermore, we let

$$
\int_{\left(x_{i}\right)}^{(0)}=1, \quad \int_{\left(x_{i}\right)}^{(m)}=\overbrace{\int_{\left(x_{i}\right)} \cdots \int_{\left(x_{i}\right)}}^{m} \text { for } 0<m \in \mathbb{Z}
$$

and denote

$$
\partial^{\alpha}=\partial_{x_{1}}^{\alpha_{1}} \partial_{x_{2}}^{\alpha_{2}} \cdots \partial_{x_{n}}^{\alpha_{n}}, \quad \int^{(\alpha)}=\int_{\left(x_{1}\right)}^{\left(\alpha_{1}\right)} \int_{\left(x_{2}\right)}^{\left(\alpha_{2}\right)} \cdots \int_{\left(x_{n}\right)}^{\left(\alpha_{n}\right)} \quad \text { for } \alpha \in \mathbb{N}^{n} .
$$

Obviously, $\int^{(\alpha)}$ is a right inverse of $\partial^{\alpha}$ for $\alpha \in \mathbb{N}^{n}$. We remark that $\int^{(\alpha)} \partial^{\alpha} \neq 1$ if $\alpha \neq 0$ due to $\partial^{\alpha}(1)=0$.

Consider the wave equation in Riemannian space with a nontrivial conformal group (cf. [I1]):

$$
u_{t t}-u_{x_{1} x_{1}}-\sum_{i, j=2}^{n} g_{i, j}\left(x_{1}-t\right) u_{x_{i} x_{j}}=0,
$$

where we assume that $g_{i, j}(z)$ are one-variable polynomials. Change variables:

$$
z_{0}=x_{1}+t, \quad z_{1}=x_{1}-t .
$$

Then

$$
\partial_{t}^{2}=\left(\partial_{z_{0}}-\partial_{z_{1}}\right)^{2}, \quad \partial_{x_{1}}^{2}=\left(\partial_{z_{0}}+\partial_{z_{1}}\right)^{2} .
$$

So the equation (2.25) changes to:

$$
2 \partial_{z_{0}} \partial_{z_{1}}+\sum_{i, j=2}^{n} g_{i, j}\left(z_{1}\right) u_{x_{i} x_{j}}=0 .
$$

Denote

$$
T_{1}=2 \partial_{z_{0}} \partial_{z_{1}}, \quad T_{2}=\sum_{i, j=2}^{n} g_{i, j}\left(z_{1}\right) \partial_{x_{i}} \partial_{x_{j}} .
$$

Take $T_{1}^{-}=\frac{1}{2} \int_{\left(z_{0}\right)} \int_{\left(z_{1}\right)}$, and

$$
\mathcal{B}=\mathbb{F}\left[z_{0}, z_{1}\right], \quad V=\mathbb{F}\left[x_{2}, \ldots, x_{n}\right], \quad V_{r}=\{f \in V \mid \operatorname{deg} f \leq r\} .
$$

Then the conditions in Lemma 2.1 hold. Thus we have:

Theorem 2.2. The space of all polynomial solutions for the equation (2.24) is:

$$
\begin{aligned}
& \operatorname{Span}\left\{\sum_{m=0}^{\infty}(-2)^{-m}\left(\sum_{i, j=2}^{n} \int_{\left(z_{0}\right)} \int_{\left(z_{1}\right)} g_{i, j}\left(z_{1}\right) \partial_{x_{i}} \partial_{x_{j}}\right)^{m}\left(f_{0} g_{0}+f_{1} g_{1}\right)\right. \\
& \left.\mid f_{0} \in \mathbb{F}\left[z_{0}\right], f_{1} \in \mathbb{F}\left[z_{1}\right], g_{0}, g_{1} \in \mathbb{F}\left[x_{2}, \ldots, x_{n}\right]\right\}
\end{aligned}
$$

with $z_{0}, z_{1}$ defined in (2.26). 
Let $m_{1}, m_{2}, \ldots, m_{n}$ be positive integers. According to Lemma 2.1, the set

$$
\begin{aligned}
& \left\{\sum_{k_{2}, \ldots, k_{n}=0}^{\infty}(-1)^{k_{2}+\cdots+k_{n}}\left(\begin{array}{c}
k_{2}+\cdots+k_{k} \\
k_{2}, \ldots, k_{n}
\end{array}\right) \int_{\left(x_{1}\right)}^{\left(\left(k_{2}+\cdots+k_{n}\right) m_{1}\right)}\left(x_{1}^{\ell_{1}}\right)\right. \\
& \left.\times \partial_{x_{2}}^{k_{2} m_{2}}\left(x_{2}^{\ell_{2}}\right) \cdots \partial_{x_{n}}^{k_{n} m_{n}}\left(x_{n}^{\ell_{n}}\right) \mid \ell_{1} \in \overline{0, m_{1}-1}, \ell_{2}, \ldots, \ell_{n} \in \mathbb{N}\right\}
\end{aligned}
$$

forms a basis of the space of polynomial solutions for the equation

$$
\left(\partial_{x_{1}}^{m_{1}}+\partial_{x_{2}}^{m_{2}}+\cdots+\partial_{x_{n}}^{m_{n}}\right)(u)=0
$$

in $\mathcal{A}$. In particular,

$$
\begin{aligned}
& \left\{\sum_{r_{2}, \ldots, r_{n}=0}^{\infty} \frac{(-1)^{r_{2}+\cdots+r_{k}}\left(\begin{array}{c}
r_{2}+\cdots+r_{n} \\
r_{2}, \ldots, r_{n}
\end{array}\right) \prod_{i=2}^{n}\left(\begin{array}{c}
\ell_{i} \\
2 r_{i}
\end{array}\right)}{\left(1+2 \epsilon\left(r_{2}+\cdots+r_{n}\right)\right)\left(\begin{array}{c}
2\left(r_{2}+\cdots+r_{n}\right) \\
2 r_{2}, \ldots, 2 r_{n}
\end{array}\right)} x_{1}^{\epsilon+2\left(r_{2}+\cdots+r_{n}\right)}\right. \\
& \left.\times x_{2}^{\ell_{2}-2 r_{2}} \cdots x_{n}^{\ell_{n}-2 r_{n}} \mid \epsilon \in\{0,1\} ; \ell_{2}, \ldots, \ell_{n} \in \mathbb{N}\right\}
\end{aligned}
$$

forms a basis of the space of harmonic polynomials in $n$ variables, that is, the space of polynomial solutions for the Laplace equation:

$$
u_{x_{1} x_{1}}+u_{x_{2} x_{2}}+\cdots+u_{x_{n} x_{n}}=0
$$

The above results can theoretically generalized as follows. Let

$$
f_{i} \in \mathbb{F}\left[x_{1}, \ldots, x_{i}\right] \quad \text { for } i \in \overline{1, n-1} \text {. }
$$

Consider the equation:

$$
\left(\partial_{x_{1}}^{m_{1}}+f_{1} \partial_{x_{2}}^{m_{2}}+\cdots+f_{n-1} \partial_{x_{n}}^{m_{n}}\right)(u)=0
$$

Denote

$$
d_{1}=\partial_{x_{1}}^{m_{1}}, \quad d_{r}=\partial_{x_{1}}^{m_{1}}+f_{1} \partial_{x_{2}}^{m_{2}}+\cdots+f_{r-1} \partial_{x_{r}}^{m_{r}} \quad \text { for } r \in \overline{2, n} .
$$

We will apply Lemma 2.1 with $T_{1}=d_{r}, T_{2}=\sum_{i=r}^{n-1} f_{i} \partial_{x_{i+1}}^{m_{i+1}}$ and $\mathcal{B}=\mathbb{F}\left[x_{1}, \ldots, x_{r}\right], V=$ $\mathbb{F}\left[x_{r+1}, \ldots, x_{n}\right]$,

$$
V_{k}=\operatorname{Span}\left\{x_{r+1}^{\ell_{r+1}} \cdots x_{n}^{\ell_{n}} \mid \ell_{s} \in \mathbb{N}, \ell_{r+1}+\sum_{i=r+2}^{n} \ell_{i}\left(\operatorname{deg} f_{r+1}+1\right) \cdots\left(\operatorname{deg} f_{i-1}+1\right) \leq k\right\}
$$

Take a right inverse $d_{1}^{-}=\int_{\left(x_{1}\right)}^{\left(m_{1}\right)}$. Suppose that we have found a right inverse $d_{s}^{-}$of $d_{s}$ for some $s \in \overline{1, n-1}$ such that

$$
x_{i} d_{s}^{-}=d_{s}^{-} x_{i}, \quad \partial_{x_{i}} d_{s}^{-}=d_{s}^{-} \partial_{x_{i}} \quad \text { for } i \in \overline{s+1, n} \text {. }
$$

Lemma 2.1 enable us to take

$$
d_{s+1}^{-}=\sum_{i=0}^{\infty}\left(-d_{s}^{-} f_{s}\right)^{i} d_{s}^{-} \partial_{x_{s+1}}^{i m_{s+1}}
$$


as a right inverse of $d_{s+1}$. Obviously,

$$
x_{i} d_{s+1}^{-}=d_{s+1}^{-} x_{i}, \quad \partial_{x_{i}} d_{s+1}^{-}=d_{s+1}^{-} \partial_{x_{i}} \quad \text { for } i \in \overline{s+2, n}
$$

according to (2.38). By induction, we have found a right inverse $d_{s}^{-}$of $d_{s}$ such that $(2.40)$ holds for each $s \in \overline{1, n}$.

We set

$$
\mathcal{S}_{r}=\left\{g \in \mathbb{F}\left[x_{1}, \ldots, x_{r}\right] \mid d_{r}(g)=0\right\} \quad \text { for } r \in \overline{1, k}
$$

By (2.38),

$$
\mathcal{S}_{1}=\sum_{i=0}^{m_{1}-1} \mathbb{F} x_{1}^{i}
$$

Suppose that we have found $\mathcal{S}_{r}$ for some $r \in \overline{1, n-1}$. Given $h \in \mathcal{S}_{r}$ and $\ell \in \mathbb{N}$, we define

$$
\sigma_{r+1, \ell}(h)=\sum_{i=0}^{\infty}\left(-d_{r}^{-} f_{r}\right)^{i}(h) \partial_{x_{r+1}}^{i m_{r+1}}\left(x_{r+1}^{\ell}\right),
$$

which is actually a finite summation. Lemma 2.1 says

$$
\mathcal{S}_{r+1}=\sum_{\ell=0}^{\infty} \sigma_{r+1, \ell}\left(\mathcal{S}_{r}\right)
$$

By induction, we obtain:

Theorem 2.3. The set

$$
\left\{\sigma_{n, \ell_{n}} \sigma_{n-1, \ell_{n-1}} \cdots \sigma_{2, \ell_{2}}\left(x_{1}^{\ell_{1}}\right) \mid \ell_{1} \in \overline{0, m_{1}-1}, \ell_{2}, \ldots, \ell_{n} \in \mathbb{N}\right\}
$$

forms a basis of the polynomial solution space $\mathcal{S}_{n}$ of the partial differential equation (2.37).

Example 2.1. Let $m_{1}, m_{2}, m_{3}, n_{1}, n_{2}$ be positive integers. Consider the following equations

$$
\partial_{x}^{m_{1}}(u)+x^{n_{1}} \partial_{y}^{m_{2}}(u)+y^{n_{2}} \partial_{z}^{m_{3}}(u)=0
$$

Now

$$
d_{1}=\partial_{x}^{m_{1}}, \quad d_{2}=\partial_{x}^{m_{1}}+x^{n_{1}} \partial_{y}^{n_{2}} .
$$

Take $d_{1}^{-}=\int_{(x)}^{\left(m_{1}\right)}$. Then

$$
\sigma_{2, \ell_{2}}\left(x^{\ell_{1}}\right)=\sum_{i=0}^{\infty}\left(-\int_{(x)}^{\left(m_{1}\right)} x^{n_{1}}\right)^{i}\left(x^{\ell_{1}}\right) \partial_{y}^{i m_{2}}\left(y^{\ell_{2}}\right) .
$$

Moreover,

$$
d_{2}^{-}=\sum_{i=0}^{\infty}\left(-\int_{(x)}^{\left(m_{1}\right)} x^{n_{1}}\right)^{i} \int_{(x)}^{\left(m_{1}\right)} \partial_{y}^{i m_{2}}
$$


by Lemma 2.1. Hence

$$
\begin{aligned}
\left(d_{2}^{-} y^{n_{2}}\right)^{k}= & \sum_{i_{1}, \ldots, i_{k}=0}^{\infty}(-1)^{i_{1}+\cdots+i_{k}}\left(\int_{(x)}^{\left(m_{1}\right)} x^{n_{1}}\right)^{i_{1}} \int_{(x)}^{m_{1}}\left(\int_{x}^{\left(m_{1}\right)} x^{n_{1}}\right)^{i_{2}} \int_{(x)}^{\left(m_{1}\right)} \cdots \\
& \left(\int_{(x)}^{\left(m_{1}\right)} x^{n_{1}}\right)^{i_{k}} \int_{(x)}^{\left(m_{1}\right)} \partial_{y}^{i_{1} m_{2}} y^{n_{2}} \partial_{y}^{i_{2} m_{2}} y^{n_{2}} \cdots \partial_{y}^{i_{k} m_{2}} y^{n_{2}} .
\end{aligned}
$$

Thus

$$
\begin{aligned}
& \sigma_{3, \ell_{3}} \sigma_{2, \ell_{2}}\left(x^{\ell_{1}}\right) \\
& =\sum_{i=0}^{\infty}\left(-\int_{(x)}^{\left(m_{1}\right)} x^{n_{1}}\right)^{i}\left(x^{\ell_{1}}\right) \partial_{y}^{i m_{2}}\left(y^{\ell_{2}}\right) z^{\ell_{3}}+\sum_{k=1}^{\infty} \sum_{i, i_{1}, \ldots, i_{k}=0}^{\infty}(-1)^{k+i+i_{1}+\cdots+i_{k}} \\
& {\left[\left(\int_{(x)}^{\left(m_{1}\right)} x^{n_{1}}\right)^{i_{1}} \int_{(x)}^{\left(m_{1}\right)}\left(\int_{(x)}^{\left(m_{1}\right)} x^{n_{1}}\right)^{i_{2}} \int_{(x)}^{\left(m_{1}\right)} \cdots\left(\int_{(x)}^{\left(m_{1}\right)} x^{n_{1}}\right)^{i_{k}} \int_{(x)}^{\left(m_{1}\right)}\left(\int_{(x)}^{\left(m_{1}\right)} x^{n_{1}}\right)^{i}\right]\left(x^{\ell_{1}}\right)} \\
& \times\left[\partial_{y}^{i_{1} m_{2}} y^{n_{2}} \partial_{y}^{i_{2} m_{2}} y^{n_{2}} \cdots \partial_{y}^{i_{k} m_{2}} y^{n_{2}} \partial_{y}^{i m_{2}}\right]\left(y^{\ell_{2}}\right) \partial_{z}^{k m_{3}}\left(z^{\ell_{3}}\right) \\
& =\sum_{i=0}^{\infty}(-1)^{i} \frac{\prod_{s=0}^{i m_{2}-1}\left(\ell_{2}-s\right)}{\prod_{r=0}^{i-1} \prod_{s=1}^{m_{1}}\left(s+n_{1}+\ell_{1}+r\left(m_{1}+n_{1}\right)\right)} x^{\ell_{1}+i\left(m_{1}+n_{1}\right)} y^{\ell_{2}-i m_{2}} \\
& +\sum_{k=1}^{\infty} \sum_{i_{0}, i_{1}, \ldots, i_{k}=0}^{\infty} \frac{(-1)^{k+i+i_{1}+\cdots+i_{k}}}{\prod_{r_{0}=0}^{i_{0}-1} \prod_{s_{0}=1}^{m_{1}}\left(s+n_{1}+\ell_{1}+r_{0}\left(m_{1}+n_{1}\right)\right)} \\
& \times \frac{\left(\prod_{p=0}^{k} \sum_{r=0}^{i_{p} m_{2}-1}\left(p n_{2}+\ell_{2}-\left(\sum_{s=0}^{p-1} i_{s}\right) m_{2}-r\right)\right)\left(\prod_{r=0}^{k m_{3}-1}\left(\ell_{3}-r\right)\right)}{\prod_{p=1}^{k}\left(\prod_{r_{p}=0}^{i_{p}} \prod_{s=1}^{m_{1}}\left(s+\ell_{1}+(p-1) m_{1}+\left(r_{p}+\sum_{q=0}^{p-1} i_{q}\right)\left(m_{1}+n_{1}\right)\right)\right)} \\
& \times x^{\left(\sum_{r=0}^{k} i_{r}\right)\left(m_{1}+n_{1}\right)+k m_{1}+\ell_{1}} y^{k n_{2}+\ell_{2}-\left(\sum_{r=0}^{k} i_{r}\right) m_{2}} z^{\ell_{3}-k m_{3}} \text {. }
\end{aligned}
$$

In order to solve the linear wave equation with dissipation and the generalized KleinGordan equation, we need the following lemma.

Lemma 2.4. Let $d=a \partial_{t}+\partial_{t}^{2}$ with $0 \neq a \in \mathbb{F}$. Take a right inverse

$$
d^{-}=\int_{(t)} \sum_{r=0}^{\infty} a^{-r-1}\left(-\partial_{t}\right)^{r}
$$

of $d$. Then

$$
\left(d^{-}\right)^{i}(1)=\frac{t^{i}}{i ! a^{i}}-\frac{t^{i-1}}{(i-2) ! a^{i+1}}+\sum_{r=2}^{i-1} \frac{(-1)^{r} \prod_{s=1}^{r-1}(i+s)}{(i-r-1) ! r ! a^{r+i}} t^{i-r} .
$$

Proof. For

$$
f(t)=\sum_{i=1}^{m} b_{i} t^{i} \in \mathbb{F}[t] t
$$

we have

$$
d(f(t))=a m b_{m} t^{m-1}+\sum_{i=1}^{m-1} i\left(a b_{i}+(i+1) b_{i+1}\right) t^{i-1} .
$$


Thus $d(f(t))=0$ if and only if $f(t) \equiv 0$. So for any given $g(t) \in \mathbb{F}[t]$, there exists a unique $f(t) \in \mathbb{F}[t] t$ such that $d(f(t))=g(t)$.

Set

$$
\xi_{a, i}(t)=\frac{t^{i}}{i ! a^{i}}-\frac{t^{i-1}}{(i-2) ! a^{i+1}}+\sum_{r=2}^{i-1} \frac{(-1)^{r} \prod_{s=1}^{r-1}(i+s)}{(i-r-1) ! r ! a^{r+i}} t^{i-r}
$$

where we treat

$$
\xi_{a, 0}(t)=1, \quad \xi_{a, 1}(t)=\frac{t}{a}, \quad \xi_{a, 2}(t)=\frac{t^{2}}{2 a^{2}}-\frac{t}{a^{3}} .
$$

Easily verify $d\left(\xi_{a, i}(t)\right)=\xi_{a, i-1}(t)$ for $i=1,2$.

Assume $i>2$. We have

$$
\begin{aligned}
& d\left(\xi_{a, i}(t)\right) \\
& =\left(a \partial_{t}+\partial_{t}^{2}\right)\left(\frac{t^{i}}{i ! a^{i}}-\frac{t^{i-1}}{(i-2) ! a^{i+1}}+\sum_{r=2}^{i-1} \frac{(-1)^{r} \prod_{s=1}^{r-1}(i+s)}{(i-r-1) ! r ! a^{r+i}} t^{i-r}\right) \\
& =\frac{t^{i-1}}{(i-1) ! a^{i-1}}-\frac{(i-1) t^{i-2}}{(i-2) ! a^{i}}+\sum_{r=2}^{i-1} \frac{(-1)^{r}(i-r) \prod_{s=1}^{r-1}(i+s)}{(i-r-1) ! r ! a^{r+i-1}} t^{i-r-1} \\
& +\frac{t^{i-2}}{(i-2) ! a^{i}}-\frac{(i-1) t^{i-3}}{(i-3) ! a^{i+1}}+\sum_{r=2}^{i-1} \frac{(-1)^{r}(i-r) \prod_{s=1}^{r-1}(i+s)}{(i-r-2) ! r ! a^{r+i}} t^{i-r-2} \\
& =\frac{t^{i-1}}{(i-1) ! a^{i-1}}-\frac{t^{i-2}}{(i-3) ! a^{i}}+\frac{(i-2)(i+1)}{(i-3) ! 2 ! a^{i+1}} t^{i-3}-\frac{(i-1) t^{i-3}}{(i-3) ! a^{i+1}} \\
& +\sum_{r=3}^{i-1}(-1)^{r}\left[\frac{(i-r) \prod_{s=1}^{r-1}(i+s)}{r !}-\frac{(i-r+1) \prod_{s=1}^{r-2}(i+s)}{(r-1) !}\right] \frac{t^{i-r-1}}{(i-r-1) ! a^{r+i-1}} \\
& =\frac{t^{i-1}}{(i-1) ! a^{i-1}}-\frac{t^{i-2}}{(i-3) ! a^{i}}+\frac{(i-2)(i+1)-2(i-1)}{(i-3) ! 2 ! a^{i+1}} t^{i-3} \\
& +\sum_{r=3}^{i-1}(-1)^{r} \frac{[(i-r)(i+r-1)-r(i-r+1)] \prod_{s=1}^{r-2}(i+s)}{(i-r-1) ! r ! a^{r+i-1}} t^{i-r-1} \\
& =\frac{t^{i-1}}{(i-1) ! a^{i-1}}-\frac{t^{i-2}}{(i-3) ! a^{i}}+\frac{i(i-3)}{(i-3) ! 2 ! a^{i+1}} t^{i-3} \\
& +\sum_{r=3}^{i-1}(-1)^{r} \frac{i(i-1-r) \prod_{s=1}^{r-2}(i+s)}{(i-r-1) ! r ! a^{r+i-1}} t^{i-r-1} \\
& =\frac{t^{i-1}}{(i-1) ! a^{i-1}}-\frac{t^{i-2}}{(i-3) ! a^{i}}+\frac{i}{(i-4) ! 2 ! a^{i+1}} t^{i-3}+\sum_{r=3}^{i-2}(-1)^{r} \frac{i \prod_{s=1}^{r-2}(i+s)}{(i-r-2) ! r ! a^{r+i-1}} t^{i-r-1} \\
& =\frac{t^{i-1}}{(i-1) ! a^{i-1}}-\frac{t^{i-2}}{(i-3) ! a^{i}}+\sum_{r=2}^{i-2}(-1)^{r} \frac{\prod_{s=1}^{r-1}(i-1+s)}{(i-r-2) ! r ! a^{r+i-1}} t^{i-r-1} \\
& =\xi_{a, i-1}(t) \text {. }
\end{aligned}
$$

Since $\left(d^{-}\right)^{0}(1)=1,\left(d^{-}\right)^{i}(1) \in \mathbb{F}[t] t$ by $(2.54)$ and $d\left[\left(d^{-}\right)^{i}(1)\right]=\left(d^{-}\right)^{i-1}(1)$ for $i \in \mathbb{N}+1$, we have $\left(d^{-}\right)^{r}(1)=\xi_{a, r}(t)$ for $r \in \mathbb{N}$ by the uniqueness, that is, (2.55) holds.

By Lemma 2.1 and the above lemma, we obtain: 
Theorem 2.5. The set

$$
\begin{aligned}
& \left\{\sum_{r_{1}, \ldots, r_{n}=0}^{\infty}\left(\begin{array}{c}
r_{1}+\cdots+r_{n} \\
r_{1}, \ldots, r_{n}
\end{array}\right)\left[\prod_{i=1}^{n}\left(2 r_{i}\right) !\left(\begin{array}{c}
\ell_{i} \\
2 r_{i}
\end{array}\right)\right]\right. \\
& \left.\times \xi_{1, r_{1}+\cdots+r_{n}}(t) x_{1}^{\ell_{1}-2 r_{1}} \cdots x_{n}^{\ell_{n}-2 r_{n}} \mid \ell_{1}, \ldots, \ell_{n} \in \mathbb{N}\right\}
\end{aligned}
$$

forms a basis of the space of polynomial solution for the linear wave equation with dissipation:

$$
u_{t t}+u_{t}-u_{x_{1} x_{1}}-u_{x_{2} x_{2}}-\cdots-u_{x_{n} x_{n}}=0
$$

Consider the following generalization of Klein-Gordan equation:

$$
u_{t t}-u_{x x}-x u_{y y}-y u_{z z}+a^{2} u=0,
$$

where $a$ is a nonzero real number. Changing variable $u=e^{a \sqrt{-1} t} v$, we get

$$
v_{t t}+2 a \sqrt{-1} v_{t}-v_{x x}-x v_{y y}-y v_{z z}=0 .
$$

We write

$$
\xi_{2 a \sqrt{-1}, i}=\zeta_{i, 0}(t)+\zeta_{i, 1}(t) \sqrt{-1}
$$

where $\zeta_{i, 0}(t)$ and $\zeta_{i, 1}(t)$ are real functions. According to (2.58),

$$
\begin{aligned}
\zeta_{2 i, 0}(t)= & (-1)^{i}\left[\frac{t^{2 i}}{(2 i) !(2 a)^{2 i}}+\sum_{r=1}^{i-1} \frac{(-1)^{r} \prod_{s=1}^{2 r-1}(2 i+s)}{(2 r) !(2(i-r)-1) !(2 a)^{2(i+r)}} t^{2(i-r)}\right], \\
& \zeta_{2 r, 1}(t)=(-1)^{i}\left[\frac{t^{2 r-1}}{(2 r-2) !(2 a)^{2 i+1}}\right. \\
& \left.+\sum_{r=1}^{i-1} \frac{(-1)^{r} \prod_{s=1}^{2 r}(2 i+s)}{(2 r+1) ![2(i-r-1)] !(2 a)^{2 i+2 r+1}} t^{2 i-2 r-1}\right], \\
& \zeta_{2 i+1,0}(t)=(-1)^{i}\left[\frac{t^{2 i}}{(2 i-1) !(2 a)^{2(i+1)}}\right. \\
& \left.+\sum_{r=1}^{i-1} \frac{(-1)^{r} \prod_{s=1}^{2 r}(2 i+s+1)}{(2 r+1) !(2 i-2 r-1) !(2 a)^{2(i+r+1)}} t^{2(i-r)}\right], \\
\zeta_{2 r+1,1}(t)=( & 1)^{i+1}\left[\frac{t^{2 i+1}}{(2 i+1) !(2 a)^{2 i+1}}+\sum_{r=1}^{i} \frac{(-1)^{r} \prod_{s=1}^{2 r-1}\left(2 i+s+(2 i-2 r) !(2 a)^{2 i+2 r+1}\right.}{(2 r)} t^{2 i-2 r+1}\right] .
\end{aligned}
$$

On the other hand, (5.22) in [X2] tells

$$
e^{t\left(\partial_{x}^{2}+x \partial_{y}^{2}+y \partial_{z}^{2}\right)}=e^{\xi_{3}} e^{\xi_{2}} e^{\xi_{1}}
$$


with

$$
\begin{aligned}
\xi_{1}= & \int_{0}^{t}\left(\partial_{x}+\int_{0}^{y_{1}}\left(\partial_{y}+y_{2} \partial_{z}^{2}\right)^{2} d y_{2}\right)^{2} d y_{1} \\
= & \int_{0}^{t}\left(\partial_{x}+y_{1} \partial_{y}^{2}+y_{1}^{2} \partial_{y} \partial_{z}^{2}+y_{1}^{3} \partial_{z}^{4} / 3\right)^{2} d y_{1} \\
= & t \partial_{x}^{2}+t^{2} \partial_{x} \partial_{y}^{2}+\frac{t^{3}}{3}\left(\partial_{y}^{4}+2 \partial_{x} \partial_{y} \partial_{z}^{2}\right)+\frac{t^{4}}{6}\left(3 \partial_{y}^{3} \partial_{z}^{2}+\partial_{x} \partial_{z}^{4}\right) \\
& +\frac{t^{5}}{3} \partial_{y}^{2} \partial_{z}^{4}+\frac{t^{6}}{9} \partial_{y} \partial_{z}^{6}+\frac{t^{7}}{21} \partial_{z}^{8}, \\
& \xi_{2}=x\left(t \partial_{y}^{2}+t^{2} \partial_{y} \partial_{z}^{2}+t^{3} \partial_{z}^{4} / 3\right), \quad \xi_{3}=t y \partial_{z}^{2} .
\end{aligned}
$$

For $\alpha=\left(\alpha_{1}, \ldots, \alpha_{11}\right) \in \mathbb{N}^{11}$, we define

$$
|\alpha|_{1}=\sum_{i=1}^{7} i \alpha_{i}+\alpha_{8}+2 \alpha_{9}+3 \alpha_{10}+\alpha_{11}, \quad \alpha !=\prod_{i=1}^{11} \alpha_{i} !
$$

and

$$
\begin{aligned}
d_{\alpha}= & \frac{|\alpha|_{1} !}{\alpha !} 2^{-\alpha_{4}} 3^{-\alpha_{3}-\alpha_{4}-\alpha_{5}-2 \alpha_{6}-\alpha_{7}-\alpha_{10}} 7^{-\alpha_{7}} x^{\alpha_{8}+\alpha_{9}+\alpha_{10}} y^{\alpha_{11}} \\
& \times \partial_{x}^{2 \alpha_{1}+\alpha_{2}} \partial_{y}^{2\left(\alpha_{2}+\alpha_{5}+\alpha_{8}\right)+\alpha_{6}+\alpha_{9}} \partial_{z}^{2\left(2 \alpha_{5}+3 \alpha_{6}+4 \alpha_{7}+\alpha_{9}+2 \alpha_{10}+\alpha_{11}\right)} \\
& \times\left(\partial_{y}^{4}+2 \partial_{x} \partial_{y} \partial_{z}^{2}\right)^{\alpha_{3}}\left(3 \partial_{y}^{3} \partial_{z}^{2}+\partial_{x} \partial_{z}^{4}\right)^{\alpha_{4}} .
\end{aligned}
$$

Then

$$
\sum_{m_{1}, m_{2}, m_{3}=0}^{\infty} \mathbb{C}\left(\sum_{\alpha \in \mathbb{N}^{11}} \zeta_{|\alpha|_{1}}(t) d_{\alpha}\left(x^{m_{1}} y^{m_{2}} z^{m_{3}}\right)\right)
$$

is the solution space in $\mathbb{C}[t, x, y, z]$ of the equation (2.64) by Lemmas 2.1 and 2.4.

Theorem 2.6. The followings are real solutions of the equation (2.63):

$$
\begin{aligned}
& u=\cos a t \sum_{\alpha \in \mathbb{N}^{11}} \zeta_{|\alpha|_{1}, 0} d_{\alpha}\left(x^{m_{1}} y^{m_{2}} z^{m_{3}}\right)-\sin a t \sum_{\alpha \in \mathbb{N}^{11}} \zeta_{|\alpha|_{1}, 1} d_{\alpha}\left(x^{m_{1}} y^{m_{2}} z^{m_{3}}\right), \\
& u=\sin a t \sum_{\alpha \in \mathbb{N}^{11}} \zeta_{|\alpha|_{1}, 0} d_{\alpha}\left(x^{m_{1}} y^{m_{2}} z^{m_{3}}\right)+\cos a t \sum_{\alpha \in \mathbb{N}^{11}} \zeta_{|\alpha|_{1}, 1} d_{\alpha}\left(x^{m_{1}} y^{m_{2}} z^{m_{3}}\right),
\end{aligned}
$$

where $m_{1}, m_{2}, m_{3} \in \mathbb{N}$.

The following lemma will be used to handle some special cases when the operator $T_{1}$ in Lemma 2.1 does not have a right inverse and to solve certain initial value problems in next section.

Lemma 2.7. Suppose that $\mathcal{A}$ is a free module over a subalgebra $\mathcal{B}$ generated by a filtrated subspace $V=\bigcup_{r=0}^{\infty} V_{r}$ (i.e., $V_{r} \subset V_{r+1}$ ). Let $T_{0}$ be a linear operator on $\mathcal{A}$ with right inverse $T_{0}^{-}$such that

$$
T_{0}(\mathcal{B}), T_{0}^{-}(\mathcal{B}) \subset \mathcal{B}, \quad T_{0}\left(\eta_{1} \eta_{2}\right)=T_{0}\left(\eta_{1}\right) \eta_{2} \quad \text { for } \eta_{1} \in \mathcal{B}, \eta_{2} \in V
$$


and let $T_{1}, \ldots, T_{m}$ be commuting linear operators on $\mathcal{A}$ such that $T_{i}(V) \subset V$,

$$
T_{0} T_{i}=T_{i} T_{0}, \quad T_{i}(f \zeta)=f T_{i}(\zeta) \quad \text { for } i \in \overline{1, m}, f \in \mathcal{B}, \zeta \in \mathcal{A} .
$$

If $T_{1}^{m}(h)=0$ with $h \in \mathcal{B}$ and $g \in V$, then

$$
u=\sum_{i=0}^{\infty}\left(\sum_{s=1}^{m}\left(T_{0}^{-}\right)^{s} T_{s}\right)^{i}(h g)=\sum_{i_{1}, \ldots, i_{m}=0}^{\infty}\left(\begin{array}{c}
i_{1}+\cdots+i_{m} \\
i_{1}, \ldots, i_{m}
\end{array}\right)\left(T_{0}^{-}\right)^{\sum_{s=1}^{m} s i_{s}}(h)\left(\prod_{r=1}^{m} T_{r}^{i_{r}}\right)(g)
$$

is a solution of the equation:

$$
\left(T_{0}^{m}-\sum_{r=1}^{m} T_{0}^{m-i} T_{i}\right)(u)=0 .
$$

Suppose

$$
T_{i}\left(V_{r}\right) \subset V_{r-1} \quad \text { for } i \in \overline{1, m}, r \in \mathbb{N},
$$

where $V_{-1}=\{0\}$. Then any polynomial solution of (2.81) is a linear combinations of the solutions of the form (2.80).

Proof. Note that

$$
T_{0}^{m-i}=T_{0}^{m}\left(T_{0}^{-}\right)^{i} \quad \text { for } i \in \overline{1, m}
$$

and

$$
\begin{aligned}
& \sum_{i_{1}+\cdots+i_{m}=i+1}\left(\begin{array}{c}
i+1 \\
i_{1}, \ldots, i_{m}
\end{array}\right) y_{1}^{i_{1}} \cdots y_{m}^{i_{m}}=\left(y_{1}+\cdots+y_{m}\right)^{i+1} \\
= & \sum_{r=1}^{m} \sum_{i_{1}+\cdots+i_{m}=i}\left(\begin{array}{c}
i \\
i_{1}, \ldots, i_{m}
\end{array}\right) y_{r} y_{1}^{i_{1}} \cdots y_{m}^{i_{m}} .
\end{aligned}
$$

Thus

$$
\begin{aligned}
& \left(T_{0}^{m}-\sum_{p=1}^{m} T_{0}^{m-p} T_{p}\right)\left[\sum_{i_{1}, \ldots, i_{m}=0}^{\infty}\left(\begin{array}{c}
i_{1}+\cdots+i_{m} \\
i_{1}, \ldots, i_{m}
\end{array}\right)\left(T_{0}^{-}\right)^{\sum_{s=1}^{m} s i_{s}}(h)\left(\prod_{r=1}^{m} T_{r}^{i_{r}}\right)(g)\right] \\
= & \sum_{i_{1}, \ldots, i_{m} \in \mathbb{N} ; i_{1}+\cdots+i_{m}>0}\left(\begin{array}{c}
i_{1}+\cdots+i_{m} \\
i_{1}, \ldots, i_{m}
\end{array}\right) T_{0}^{m}\left(T_{0}^{-}\right)^{\sum_{s=1}^{m} s i_{s}}(h)\left(\prod_{r=1}^{m} T_{r}^{i_{r}}\right)(g) \\
& -\sum_{i_{1}, \ldots, i_{m}=0}^{\infty} \sum_{p=1}^{m}\left(\begin{array}{c}
i_{1}+\cdots+i_{m} \\
i_{1}, \ldots, i_{m}
\end{array}\right) T_{0}^{m}\left(T_{0}^{-}\right)^{i_{p}+\sum_{s=1}^{m} s i_{s}}(h)\left(T_{p} \prod_{r=1}^{m} T_{r}^{i_{r}}\right)(g)=0 .
\end{aligned}
$$

Suppose that (2.82) holds. Let $u \in \mathcal{B} V_{k} \backslash \mathcal{B} V_{k-1}$ be a solution of (2.81). Take a basis $\left\{\phi_{i}+V_{k-1} \mid i \in I\right\}$ of $V_{k} / V_{k-1}$. Write

$$
u=\sum_{i \in I} h_{i} \phi_{i}+u^{\prime}, \quad h_{i} \in \mathcal{B}, u^{\prime} \in \mathcal{B} V_{k-1} .
$$

Since

$$
T_{r}\left(\phi_{i}\right) \in V_{k-1} \quad \text { for } i \in I, r \in \overline{1, m}
$$


by (2.82), we have

$$
\left(T_{0}^{m}-\sum_{r=1}^{m} T_{0}^{m-i} T_{i}\right)(u) \equiv \sum_{i \in I} T_{0}^{m}\left(h_{i}\right) \phi_{i} \equiv 0\left(\bmod \mathcal{B} V_{k-1}\right) .
$$

Hence

$$
T_{0}^{m}\left(h_{i}\right)=0 \quad \text { for } i \in I
$$

Now

$$
u-\sum_{j \in I} \sum_{i_{1}, \ldots, i_{m}=0}^{\infty}\left(\begin{array}{c}
i_{1}+\cdots+i_{m} \\
i_{1}, \ldots, i_{m}
\end{array}\right)\left(T_{0}^{-}\right)^{\sum_{s=1}^{m} s i_{s}}\left(h_{j}\right)\left(\prod_{r=1}^{m} T_{r}^{i_{r}}\right)\left(\phi_{j}\right) \in \mathcal{B} V_{k-1}
$$

is a solution of (2.81). By induction on $k, u$ is a linear combinations of the solutions of the form (2.80).

We remark that the above lemma does not imply Lemma 2.1 because $T_{1}$ and $T_{2}$ in Lemma 2.1 may not commute.

Let $d_{1}$ be a differential operator on $\mathbb{F}\left[x_{1}, x_{2}, \ldots, x_{r}\right]$ and let $d_{2}$ be a locally nilpotent differential operator on $V=\mathbb{F}\left[x_{r+1}, \ldots, x_{n}\right]$. Set

$$
V_{i}=\left\{f \in V \mid d_{2}^{i}(f) \neq 0, d_{2}^{i+1}(f)=0\right\} \quad \text { for } i \in \mathbb{N} .
$$

Take a subset $\left\{\psi_{i, j} \mid i \in \mathbb{N}+1, j \in I_{i}\right\}$ of $V$ such that $\left\{\psi_{i, j}+V_{i-1} \mid j \in I_{i}\right\}$ forms a basis of $V_{i} / V_{i-1}$ for $i \in \mathbb{N}+1$. Fix $h \in \mathbb{F}\left[x_{1}, \ldots, x_{r}\right]$.

Lemma 2.8. Let $i$ be a positive integer. Suppose that

$$
u=\sum_{j \in I_{i}} f_{j} \psi_{i, j}+u^{\prime} \in \mathbb{F}\left[x_{1}, x_{2}, \ldots, x_{n}\right]
$$

with $f_{j} \in \mathbb{F}\left[x_{1}, \ldots, x_{r}\right]$ and $d_{2}^{i}\left(u^{\prime}\right)=0$ is a solution of the equation:

$$
\left(d_{1}-h d_{2}\right)(u)=0
$$

Then the system

$$
\xi_{0}=f_{j}, \quad d_{1}\left(\xi_{s+1}\right)=h \xi_{s} \quad \text { for } s \in \overline{0, i-1}
$$

has a solution $\xi_{1}, \ldots, \xi_{i} \in \mathbb{F}\left[x_{1}, \ldots, x_{r}\right]$ for each $j \in I_{i}$.

Proof. Denote $V_{-1}=\{0\}$. Observe that if $\left\{f_{j}+V_{p} \mid p \in J\right\}$ is a linearly independent subset of $V_{p+1} / V_{p}$, then $\left\{d_{2}^{s}\left(f_{j}\right)+V_{p-s} \mid p \in J\right\}$ is a linearly independent subset of $V_{p-s+1} / V_{p-s}$ for $s \in \overline{1, p+1}$ by (2.91). By induction, we take a subset $\left\{\phi_{i-s, j} \mid j \in J_{i-s}\right\}$ of $V_{i-s}$ for each $s \in \overline{1, i}$ such that

$$
\left\{d_{2}^{s}\left(\psi_{i, j_{1}}\right)+V_{i-s-1}, d_{2}^{s-p}\left(\phi_{i-p, j_{2}}\right)+V_{i-s-1} \mid p \in \overline{1, s}, j_{1} \in I_{i}, j_{2} \in J_{i-p}\right\}
$$

forms a basis of $V_{i-s} / V_{i-s-1}$ for $s \in \overline{1, i}$. Denote

$$
\mathcal{B}=\sum_{s=1}^{i} \sum_{p=0}^{i-s} \sum_{j \in J_{i-s}} \mathbb{F}\left[x_{1}, \ldots, x_{r}\right] d_{2}^{p}\left(\phi_{i-s, j}\right)
$$


Now we write

$$
u=\sum_{j \in I_{i}}\left[f_{j} \psi_{i, j}+\sum_{s=1}^{i} f_{s, j} d^{s}\left(\psi_{i, j}\right)\right]+v, \quad v \in \mathcal{B}, f_{s, j} \in \mathbb{F}\left[x_{1}, \ldots, x_{r}\right] .
$$

Then (2.93) becomes

$$
\begin{aligned}
& \sum_{j \in I_{i}}\left[d_{1}\left(f_{j}\right) \psi_{i, j}+\left(d_{1}\left(f_{1, j}\right)-h f_{j}\right) d_{2}\left(\psi_{i, j}\right)+\sum_{s=2}^{i}\left(d_{1}\left(f_{s, j}\right)-h f_{s-1, j}\right) d^{s}\left(\psi_{i, j}\right)\right] \\
& +\left(d_{1}-h d_{2}\right)(v)=0 .
\end{aligned}
$$

Since $\left(d_{1}-h d_{2}\right)(v) \in \mathcal{B}$, we have:

$$
d_{1}\left(f_{j}\right)=0, \quad d_{1}\left(f_{1, j}\right)=h f_{j}, \quad d_{1}\left(f_{s, j}\right)=h f_{s-1, j}
$$

for $j \in I_{i}$ and $s \in \overline{2, i}$. So (2.94) has a solution $\xi_{1}, \ldots, \xi_{i} \in \mathbb{F}\left[x_{1}, \ldots, x_{r}\right]$ for each $j \in I_{i}$.

Set

$$
\mathcal{S}_{0}=\left\{f \in \mathbb{F}\left[x_{1}, \ldots, x_{r}\right] \mid d_{1}(f)=0\right\}
$$

and

$$
\mathcal{S}_{i}=\left\{f_{0} \in \mathcal{S}_{0} \mid d_{1}\left(f_{s}\right)=h f_{s-1} \text { for some } f_{1}, \ldots, f_{i} \in \mathbb{F}\left[x_{1}, \ldots, x_{r}\right]\right\}
$$

for $i \in \mathbb{N}+1$. For each $i \in \mathbb{N}+1$ and $f \in \mathcal{S}_{i}$, we fix $\left\{\sigma_{1}(f), \ldots, \sigma_{i}(f)\right\} \subset \mathbb{F}\left[x_{1}, \ldots, x_{r}\right]$ such that

$$
d_{1}\left(\sigma_{1}(f)\right)=f, \quad d_{1}\left(\sigma_{s}(f)\right)=\sigma_{s-1}(f) \quad \text { for } s \in \overline{2, i} .
$$

Denote $\sigma_{0}(f)=f$. By the proof of Lemma 2.1 and the above Lemma, we obtain:

Lemma 2.9. The set

$$
\sum_{i=0}^{\infty} \sum_{j \in I_{i}} \sum_{f \in \mathcal{S}_{i}} \mathbb{F}\left(\sum_{s=0}^{i} \sigma_{s}(f) d_{2}^{s}\left(\psi_{i, j}\right)\right)
$$

is the solution space of the equation (2.93) in $\mathbb{F}\left[x_{1}, x_{2}, \ldots, x_{n}\right]$.

Let $\epsilon \in\{1,-1\}$ and let $\lambda$ be a nonzero real number. Next we want to find all the polynomial solutions of the equation:

$$
u_{t t}+\frac{\lambda}{t} u_{t}-\epsilon\left(u_{x_{1} x_{1}}+u_{x_{2} x_{2}}+\cdots+u_{x_{n} x_{n}}\right)=0
$$

which is the generalized anisymmetrical Laplace equation (cf. [A]) if $\epsilon=-1$. Rewrite the above equation as:

$$
t u_{t t}+\lambda u_{t}-\epsilon t\left(u_{x_{1} x_{1}}+u_{x_{2} x_{2}}+\cdots+u_{x_{n} x_{n}}\right)=0 .
$$

Set

$$
d=t \partial_{t}^{2}+\lambda \partial_{t}
$$


Denote

$$
\mathcal{S}=\{f \in \mathbb{F}[t] \mid d(f)=0\}
$$

Note that

$$
d\left(t^{m}\right)=m(\lambda+m-1) t^{m-1} \quad \text { for } m \in \mathbb{N} .
$$

So

$$
\mathcal{S}= \begin{cases}\mathbb{F} & \text { if } \lambda \notin-(\mathbb{N}+1), \\ \mathbb{F}+\mathbb{F} t^{-\lambda+1} & \text { if } \lambda \in-(\mathbb{N}+1) .\end{cases}
$$

In particular, $t^{-\lambda} \notin d(\mathbb{F}[t])$ and so $d$ does not have a right inverse when $\lambda$ is a negative integer.

Set

$$
\phi_{0}(t)=1, \quad \phi_{i}(t)=\frac{t^{2 i}}{i ! 2^{i} \prod_{r=0}^{i-1}(\lambda+2 r+1)}
$$

for $i \in \mathbb{N}+1$ and when $\lambda \neq-1,-3, \ldots,-(2 i-1)$. When $\lambda \in-(\mathbb{N}+1)$, we set

$$
\psi_{0}=t^{1-\lambda}, \quad \psi_{i}=\frac{t^{2 i+1-\lambda}}{2^{i} i ! \prod_{r=1}^{i}(2 r+1-\lambda)} \quad \text { for } i \in \mathbb{N}+1 \text {. }
$$

Define

$$
V=\mathbb{F}\left[x_{1}, x_{2}, \ldots, x_{n}\right], \quad \Delta_{n}=\sum_{r=1}^{n} \partial_{x_{r}}^{2}, \quad \Delta_{2, n}=\sum_{s=2}^{n} \partial_{x_{s}}^{2}
$$

and

$$
V_{i}=\left\{f \in V \mid \Delta_{n}^{i}(f)=0\right\} \quad \text { for } i \in \mathbb{N}+1 \text {. }
$$

Observe

$$
\begin{aligned}
& \sum_{j_{1}, \ldots, j_{i}=0}^{\infty}(-1)^{j_{1}+\cdots+j_{i}}\left(\begin{array}{c}
j_{1}+\cdots+j_{i} \\
j_{1}, \ldots, j_{i}
\end{array}\right) \prod_{r=1}^{i}\left[\left(\begin{array}{c}
i \\
r
\end{array}\right) t^{r}\right]^{j_{r}}=\sum_{p=0}^{\infty}\left(-\sum_{s=1}^{i}\left(\begin{array}{l}
i \\
s
\end{array}\right) t^{i}\right)^{p} \\
= & \frac{1}{(1+t)^{i}}=\sum_{r=0}^{\infty}(-1)^{r}\left(\begin{array}{c}
i+r-1 \\
r
\end{array}\right) t^{r}
\end{aligned}
$$

for $|t|<1$. Applying Lemma 2.7 to $\Delta_{n}^{i}=\sum_{r=0}^{i}\left(\begin{array}{l}i \\ r\end{array}\right) \partial_{x_{1}}^{2(i-r)} \Delta_{2, n}^{r}$ with $m=i, T_{0}=\partial_{x_{1}}^{2}$ and $T_{r}=-\left(\begin{array}{c}i \\ r\end{array}\right) \Delta_{2, n}^{r}$ for $r \in \overline{1, i}$, we get a basis

$$
\left\{\sum_{r=0}^{\infty}(-1)^{r}\left(\begin{array}{c}
i+r-1 \\
r
\end{array}\right) \frac{x_{1}^{\ell_{1}+2 r}}{\left(\ell_{1}+2 r\right) !} \Delta_{2, n}^{r}\left(x_{2}^{\ell_{2}} \cdots x_{n}^{\ell_{n}}\right) \mid \ell_{1} \in \overline{0,2 i-1}, \ell_{2}, \ldots, \ell_{n} \in \mathbb{N}\right\}
$$

of $V_{i}$. Hence we obtain:

Theorem 2.10. If $\lambda \notin-(\mathbb{N}+1)$, then the set

$$
\left\{\sum_{r=0}^{\infty} \epsilon^{r} \phi_{r}(t) \Delta_{n}^{r}\left(x_{1}^{\ell_{1}} \cdots x_{n}^{\ell_{n}}\right) \mid \ell_{1}, \ldots, \ell_{n} \in \mathbb{N}\right\}
$$

forms a basis of the space of the polynomial solutions for the equation (2.104). When $\lambda$ is a negative even integer, the set

$$
\left\{\sum_{r=0}^{\infty} \epsilon^{r} \phi_{r}(t) \Delta_{n}^{n}\left(x_{1}^{\ell_{1}} \cdots x_{n}^{\ell_{n}}\right), \sum_{r=0}^{\infty} \epsilon^{r} \psi_{r}(t) \Delta_{n}^{n}\left(x_{1}^{\ell_{1}} \cdots x_{n}^{\ell_{n}}\right) \mid \ell_{1}, \ldots, \ell_{n} \in \mathbb{N}\right\}
$$


forms a basis of the space of the polynomial solutions for the equation (2.104). Assume that $\lambda=-2 k-1$ is a negative odd integer. The set

$$
\begin{aligned}
& \left\{\sum_{s=0}^{k} \sum_{r=0}^{\infty}(-1)^{r} \epsilon^{s}\left(\begin{array}{c}
k+r-1 \\
r
\end{array}\right) \phi_{s}(t) \Delta_{n}^{s}\left[\frac{x_{1}^{\ell_{1}+2 r}}{\left(\ell_{1}+2 r\right) !} \Delta_{2, n}^{r}\left(x_{2}^{\ell_{2}} \cdots x_{n}^{\ell_{n}}\right)\right],\right. \\
& \left.\sum_{r=0}^{\infty} \epsilon^{r} \psi_{r}(t) \Delta_{n}^{r}\left(x_{1}^{\ell_{1}^{\prime}} x_{2}^{\ell_{2}} \cdots x_{n}^{\ell_{n}}\right) \mid \ell_{1} \in \overline{0,2 i-1}, \ell_{1}^{\prime}, \ell_{2}, \ldots, \ell_{n} \in \mathbb{N}\right\}
\end{aligned}
$$

is a basis of the space of the polynomial solutions for the equation (2.104).

Finally, we consider the special Euler-Poisson-Darboux equation:

$$
u_{t t}-u_{x_{1} x_{1}}-u_{x_{2} x_{2}}-\cdots-u_{x_{n} x_{n}}-\frac{m(m+1)}{t^{2}} u=0
$$

with $m \neq-1,0$. Change the equations to:

$$
t^{2} u_{t t}-t^{2}\left(u_{x_{1} x_{1}}+u_{x_{2} x_{2}}+\cdots+u_{x_{n} x_{n}}\right)-m(m+1) u=0 .
$$

Letting $u=t^{m+1} v$, we have:

$$
t^{2} u_{t t}=m(m+1) t^{m+1} v+2(m+1) t^{m+2} v_{t}+t^{m+3} v_{t t}
$$

Substituting (2.121) into (2.120), we get

$$
t v_{t t}+2(m+1) v_{t}-t\left(v_{x_{1} x_{1}}+v_{x_{2} x_{2}}+\cdots+v_{x_{n} x_{n}}\right)=0
$$

If we change variable $u=t^{-m} v$, then the equation (2.120) becomes

$$
t v_{t t}-2 m v_{t}-t\left(v_{x_{1} x_{1}}+v_{x_{2} x_{2}}+\cdots++_{x_{n} x_{n}}\right)=0
$$

Equations (2.121) and (2.122) are special cases of the equation (2.105) with $\epsilon=1$, and $\lambda=2(m+1)$ and $\lambda=-2 m$, respectively.

\section{Initial Value Problems}

In this section, we will solve two initial value problems.

Let $m$ and $n>1$ be positive integers and let

$$
f_{i}\left(\partial_{x_{2}}, \ldots, \partial_{x_{n}}\right) \in \mathbb{R}\left[\partial_{x_{2}}, \ldots, \partial_{x_{n}}\right] \quad \text { for } i \in \overline{1, m}
$$

We want to solve the equation:

$$
\left(\partial_{x_{1}}^{m}-\sum_{r=1}^{m} \partial_{x_{1}}^{m-i} f_{i}\left(\partial_{x_{2}}, \ldots, \partial_{x_{n}}\right)\right)(u)=0
$$

with $x_{1} \in \mathbb{R}$ and $x_{r} \in\left[-a_{r}, a_{r}\right]$ for $r \in \overline{2, n}$, subject to the condition

$$
\partial_{x_{1}}^{s}(u)\left(0, x_{2}, \ldots, x_{n}\right)=g_{s}\left(x_{2}, \ldots, x_{n}\right) \quad \text { for } s \in \overline{0, m-1}
$$


where $a_{2}, \ldots, a_{n}$ are positive real numbers and $g_{0}, \ldots, g_{m-1}$ are continuous functions. For convenience, we denote

$$
k_{i}^{\dagger}=\frac{k_{i}}{a_{i}}, \quad \vec{k}^{\dagger}=\left(k_{2}^{\dagger}, \ldots, k_{n}^{\dagger}\right) \quad \text { for } \vec{k}=\left(k_{2}, \ldots, k_{n}\right) \in \mathbb{N}^{n-1} .
$$

Set

$$
e^{2 \pi\left(\vec{k}^{\dagger} \cdot \vec{x}\right) \sqrt{-1}}=e^{\sum_{r=2}^{n} 2 \pi k_{r}^{\dagger} x_{r} \sqrt{-1}}
$$

For $r \in \overline{0, m-1}$,

$$
\begin{aligned}
& \frac{1}{r !} \sum_{i_{1}, \ldots, i_{m}=0}^{\infty}\left(\begin{array}{c}
i_{1}+\cdots+i_{m} \\
i_{1}, \ldots, i_{m}
\end{array}\right) \int_{\left(x_{1}\right)}^{\left(\sum_{s=1}^{m} s i_{s}\right)}\left(x_{1}^{r}\right)\left(\prod_{p=1}^{m} f_{p}\left(\partial_{x_{2}}, \ldots, \partial_{x_{n}}\right)^{i_{p}}\right)\left(e^{2 \pi\left(\vec{k}^{\dagger} \cdot \vec{x}\right) \sqrt{-1}}\right) \\
= & \sum_{i_{1}, \ldots, i_{m}=0}^{\infty}\left(\begin{array}{c}
i_{1}+\cdots+i_{m} \\
i_{1}, \ldots, i_{m}
\end{array}\right) \frac{x_{1}^{r+\sum_{s=1}^{m} s i_{s}}}{\left(r+\sum_{s=1}^{m} s i_{s}\right) !} \\
& \times\left[\prod_{p=1}^{m} f_{p}\left(2 k_{2}^{\dagger} \pi \sqrt{-1}, \ldots, 2 k_{n}^{\dagger} \pi \sqrt{-1}\right)^{i_{p}}\right] e^{2 \pi\left(\vec{k}^{\dagger} \cdot \vec{x}\right) \sqrt{-1}}
\end{aligned}
$$

is a complex solution of the equation (3.2) by Lemma 2.7 for any $\vec{k} \in \mathbb{Z}^{n-1}$. We write

$$
\begin{aligned}
& \sum_{i_{1}, \ldots, i_{m}=0}^{\infty}\left(\begin{array}{c}
i_{1}+\cdots+i_{m} \\
i_{1}, \ldots, i_{m}
\end{array}\right) \frac{x_{1}^{r} \prod_{p=1}^{m}\left(x_{1}^{p} f_{p}\left(2 k_{2}^{\dagger} \pi \sqrt{-1}, \ldots, 2 k_{n}^{\dagger} \pi \sqrt{-1}\right)\right)^{i_{p}}}{\left(r+\sum_{s=1}^{m} s i_{s}\right) !} \\
= & \phi_{r}\left(x_{1}, \vec{k}\right)+\psi_{r}\left(x_{1}, \vec{k}\right) \sqrt{-1},
\end{aligned}
$$

where $\phi_{r}\left(x_{1}, \vec{k}\right)$ and $\psi_{r}\left(x_{1}, \vec{k}\right)$ are real functions. Moreover,

$$
\partial_{x_{1}}^{s}\left(\phi_{r}\right)(0, \vec{k})=\delta_{r, s}, \quad \partial_{x_{1}}^{s}\left(\psi_{r}\right)(0, \vec{k})=0 \quad \text { for } s \in \overline{0, r} .
$$

We define $\overrightarrow{0} \prec \vec{k}$ if its first nonzero coordinate is a positive integer. By superposition principle and Fourier expansions, we get:

Theorem 3.1. The solution of the equation (3.2) subject to the condition (3.3) is:

$$
\begin{aligned}
u= & \sum_{r=0}^{m-1} \sum_{\overrightarrow{0} \preceq \vec{k} \in \mathbb{Z}^{n-1}}\left[b_{r}(\vec{k})\left(\phi_{r}\left(x_{1}, \vec{k}^{\dagger}\right) \cos 2 \pi\left(\vec{k}^{\dagger} \cdot \vec{x}\right)-\psi_{r}\left(x_{1}, \vec{k}^{\dagger}\right) \sin 2 \pi\left(\vec{k}^{\dagger} \cdot \vec{x}\right)\right)\right. \\
& \left.+c_{r}(\vec{k})\left(\phi_{r}\left(x_{1}, \vec{k}^{\dagger}\right) \sin 2 \pi\left(\vec{k}^{\dagger} \cdot \vec{x}\right)+\psi_{r}\left(x_{1}, \vec{k}^{\dagger}\right) \cos 2 \pi\left(\vec{k}^{\dagger} \cdot \vec{x}\right)\right)\right]
\end{aligned}
$$

with

$$
\begin{aligned}
b_{r}(\vec{k})= & \frac{1}{2^{n-2} a_{2} \cdots a_{n}} \int_{-a_{2}}^{a_{2}} \cdots \int_{-a_{n}}^{a_{n}} g_{r}\left(x_{2}, \ldots, x_{n}\right) \cos 2 \pi\left(\vec{k}^{\dagger} \cdot \vec{x}\right) d x_{n} \cdots d x_{2} \\
& -\sum_{s=0}^{r-1}\left(b_{s}(\vec{k}) \partial_{x_{1}}^{r}\left(\phi_{s}\right)(0, \vec{k})+c_{s}(\vec{k}) \partial_{x_{1}}^{r}\left(\psi_{s}\right)(0, \vec{k})\right)
\end{aligned}
$$

and

$$
\begin{aligned}
c_{r}(\vec{k})= & \frac{1}{2^{n-2} a_{2} \cdots a_{n}} \int_{-a_{2}}^{a_{2}} \cdots \int_{-a_{n}}^{a_{n}} g_{r}\left(x_{2}, \ldots, x_{n}\right) \sin 2 \pi\left(\vec{k}^{\dagger} \cdot \vec{x}\right) d x_{n} \cdots d x_{2} \\
& -\sum_{s=0}^{r-1}\left(c_{s}(\vec{k}) \partial_{x_{1}}^{r}\left(\phi_{s}\right)(0, \vec{k})-b_{s}(\vec{k}) \partial_{x_{1}}^{r}\left(\psi_{s}\right)(0, \vec{k})\right) .
\end{aligned}
$$


The convergence of the series (3.9) is guaranteed by the Kovalevskaya Theorem on the existence and uniqueness of the solution of linear partial differential equations when the functions in (3.3) are analytic.

Remark 3.2. (1) If we take $f_{i}=b_{i}$ with $i \in \overline{1, m}$ to be constant functions and $\vec{k}=\overrightarrow{0}$ in (3.6), we get $m$ fundamental solutions

$$
\varphi_{r}(x)=\sum_{i_{1}, \ldots, i_{m}=0}^{\infty}\left(\begin{array}{c}
i_{1}+\cdots+i_{m} \\
i_{1}, \ldots, i_{m}
\end{array}\right) \frac{x^{r} \prod_{p=1}^{m}\left(b_{p} x^{p}\right)^{i_{p}}}{\left(r+\sum_{s=1}^{m} s i_{s}\right) !}, \quad r \in \overline{0, m-1},
$$

of the constant-coefficient ordinary differential equation

$$
y^{(m)}-b_{1} y^{(m-1)}-\cdots-b_{m-1} y^{\prime}-b_{m}=0 .
$$

Given the initial conditions:

$$
y^{(r)}(0)=c_{r} \quad \text { for } r \in \overline{0, m-1}
$$

we define $a_{0}=c_{0}$ and

$$
a_{r}=c_{r}-\sum_{s=0}^{r-1} \sum_{i_{1}, \ldots, i_{r-s} \in \mathbb{N} ; \sum_{p=1}^{r} p i_{p}=r-s}\left(\begin{array}{c}
r-s \\
i_{1}, \ldots, i_{r-s}
\end{array}\right) a_{s} b_{1}^{i_{1}} \cdots b_{r-s}^{i_{r-s}}
$$

by induction on $r \in \overline{1, m-1}$. Now the solution of (3.13) subject to the condition (3.14) is exactly

$$
y=\sum_{r=0}^{m-1} a_{r} \varphi_{r}(x)
$$

From the above results, it seems that the following functions

$$
\mathcal{Y}_{r}\left(y_{1}, \ldots, y_{m}\right)=\sum_{i_{1}, \ldots, i_{m}=0}^{\infty}\left(\begin{array}{c}
i_{1}+\cdots+i_{m} \\
i_{1}, \ldots, i_{m}
\end{array}\right) \frac{y_{1}^{i_{1}} y_{2}^{i_{2}} \cdots y_{m}^{i_{m}}}{\left(r+\sum_{s=1}^{m} s i_{s}\right) !} \quad \text { for } \quad r \in \mathbb{N}
$$

are important natural functions. Indeed,

$$
\begin{gathered}
\mathcal{Y}_{1}(x)=e^{x}, \mathcal{Y}_{0}(0,-x)=\cos x, \mathcal{Y}_{1}(0,-x)=\frac{\sin x}{x} \\
\varphi_{r}(x)=x^{r} \mathcal{Y}_{r}\left(b_{1} x, b_{2} x^{2}, \ldots, b_{m} x^{m}\right)
\end{gathered}
$$

and

$$
\begin{aligned}
& \phi_{r}\left(x_{1}, \vec{x}\right)+\psi_{r}\left(x_{1}, \vec{x}\right) \sqrt{-1} \\
= & \left.x_{1}^{r} \mathcal{Y}_{r}\left(x_{1} f_{1}\left(2 k_{2}^{\dagger} \pi \sqrt{-1}, \ldots, 2 k_{n}^{\dagger} \pi \sqrt{-1}\right)\right), \ldots, x_{1}^{m} f_{m}\left(2 k_{2}^{\dagger} \pi \sqrt{-1}, \ldots, 2 k_{n}^{\dagger} \pi \sqrt{-1}\right)\right)
\end{aligned}
$$

for $r \in \overline{0, m}$.

(2) We can solve the initial value problem (3.2) and (3.3) with the constant-coefficient differential operators $f_{i}\left(\partial_{2}, \ldots, \partial_{n}\right)$ replaced by variable-coefficient differential operators 
$\phi_{i}\left(\partial_{2}, \ldots, \partial_{n_{1}}\right) \psi_{i}\left(x_{n_{1}+1}, \ldots, x_{n}\right)$ for some $2<n_{1}<n$, where $\phi_{i}\left(\partial_{2}, \ldots, \partial_{n_{1}}\right)$ are polynomials in $\partial_{2}, \ldots, \partial_{n_{1}}$ and $\psi\left(x_{n_{1}+1}, \ldots, x_{n}\right)$ are polynomials in $x_{n_{1}+1}, \ldots, x_{n}$.

Let $\mathcal{T}=(\mathcal{N}, \mathcal{E})$ be a tree with $n$ nodes. Now we consider the following generalized wave equation

$$
u_{t t}-d_{\mathcal{T}}(u)=0
$$

(cf. (1.6)) with $t \in \mathbb{R}$ and $x_{r} \in\left[-a_{r}, a_{r}\right]$ for $r \in \overline{1, n}$ subject to the condition

$$
u\left(0, x_{1}, \ldots, x_{n}\right)=g_{0}\left(x_{1}, \ldots, x_{n}\right), u_{t}\left(0, x_{1}, \ldots, x_{n}\right)=g_{1}\left(x_{1}, \ldots, x_{n}\right)
$$

Denote

$$
\vec{x}=\left(x_{1}, \ldots, x_{n}\right), \quad k_{i}^{\dagger}=\frac{k_{i}}{a_{i}}, \vec{k}^{\dagger}=\left(k_{1}^{\dagger}, \ldots, k_{n}^{\dagger}\right) \quad \text { for } \vec{k}=\left(k_{1}, \ldots, k_{n}\right) \in \mathbb{N}^{n} .
$$

For $\epsilon \in\{0,1\}$,

$$
\sum_{i=0}^{\infty} \int_{(t)}^{(2 i)}\left(t^{\epsilon}\right) d_{\mathcal{T}}^{i}\left(e^{2 \pi\left(\vec{k}^{\dagger} \cdot \vec{x}\right) \sqrt{-1}}\right)
$$

are solutions of (3.21) by Lemma 2.1. Moreover,

$$
\sum_{i=0}^{\infty} \int_{(t)}^{(2 i)}(1) d_{\mathcal{T}}^{i}=\frac{1}{2}\left(e^{t d_{\mathcal{T}}}+e^{-t d_{\mathcal{T}}}\right), \sum_{i=0}^{\infty} \int_{(t)}^{(2 i)}(t) d_{\mathcal{T}}^{i}=\frac{1}{2} \int_{(t)}\left(e^{t d_{\mathcal{T}}}+e^{-t d_{\mathcal{T}}}\right) .
$$

Recall that $\Psi$ is the set of all tips in $\mathcal{T}=(\mathcal{N}, \mathcal{E})$. A node $\iota_{j}$ is called a descendant of $\iota_{i}$ if $i<j$ and there exist a sequence

$$
i_{0}=i<i_{1}<\cdots i_{r-1}<i_{r}=j
$$

such that

$$
\left(\iota_{i_{r}}, \iota_{r+1}\right) \in \mathcal{E} \quad \text { for } r \in \overline{0, r-1}
$$

Set

$$
\mathcal{D}_{i}=\text { the set of all descendants of } \iota_{i} \text {. }
$$

Let

$$
\tilde{\xi}_{r}(t)=t \partial_{x_{r}}^{m_{r}} \quad \text { for } \quad \iota_{r} \in \Psi .
$$

Suppose that we have defined $\left\{\tilde{\xi}_{s}(t) \mid \iota_{s} \in \mathcal{D}_{i}\right\}$. Denote

$$
\Theta_{i}=\left\{\iota_{s} \in \mathcal{N} \mid\left(\iota_{i}, \iota_{s}\right) \in \mathcal{E}\right\} \subset \mathcal{D}_{i} .
$$

Now we define

$$
\tilde{\xi}_{i}(t)=\int_{0}^{t}\left(\partial_{x_{i}}+\sum_{\iota_{s} \in \Theta_{i}} \tilde{\xi}_{s}\left(y_{i}\right)\right)^{2} d y_{i}
$$

By induction, we have defined all $\left\{\tilde{\xi}_{1}(t), \ldots, \tilde{\xi}_{n}(t)\right\}$. Moreover, we let

$$
\xi_{1}\left(t, \partial_{\xi_{1}}, \ldots, \partial_{x_{n}}\right)=\tilde{\xi}_{1}(t), \quad \xi_{i}\left(t, \partial_{\xi_{1}}, \ldots, \partial_{x_{n}}\right)=x_{p(i)} \tilde{\xi}_{i}(t)
$$


for $i \in \overline{2, n}$, where $\iota_{p(i)}$ is the unique (parent) node such that $\left(\iota_{p(i)}, \iota_{i}\right) \in \mathcal{E}$. According to $(5.48)$ in $[\mathrm{X} 2]$,

$$
e^{t d_{\mathcal{T}}}=e^{\xi_{n}\left(t, \partial_{\xi_{1}}, \ldots, \partial_{x_{n}}\right)} e^{\xi_{n-1}\left(t, \partial_{\xi_{1}}, \ldots, \partial_{x_{n}}\right)} \cdots e^{\xi_{1}\left(t, \partial_{\xi_{1}}, \ldots, \partial_{x_{n}}\right)}
$$

In fact,

$$
e^{t d_{\mathcal{T}}}\left(e^{2 \pi\left(\vec{k}^{\dagger} \cdot \vec{x}\right) \sqrt{-1}}\right)=e^{\sum_{i=1}^{n} \xi_{i}\left(t, 2 \pi k_{1}^{\dagger} \sqrt{-1}, \ldots, 2 \pi k_{n}^{\dagger} \sqrt{-1}\right)} e^{2 \pi\left(\vec{k}^{\dagger} \cdot \vec{x}\right) \sqrt{-1}}
$$

Define

$$
\begin{aligned}
& \phi_{\vec{k}}\left(t, x_{1}, \ldots, x_{n}\right)=\frac{1}{4}\left(e^{\left.2 \pi \vec{k} k^{\dagger} \cdot \vec{x}\right) \sqrt{-1}+\sum_{i=1}^{n} \xi_{i}\left(t, 2 \pi k_{1}^{\dagger} \sqrt{-1}, \ldots, 2 \pi k_{n}^{\dagger} \sqrt{-1}\right)}\right. \\
& +e^{2 \pi\left(k^{\dagger} \cdot \vec{x}\right) \sqrt{-1}-\sum_{i=1}^{n} \xi_{i}\left(t, 2 \pi k_{1}^{\dagger} \sqrt{-1}, \ldots, 2 \pi k_{n}^{\dagger} \sqrt{-1}\right)} \\
& +e^{-2 \pi\left(\vec{k}^{\dagger} \cdot \vec{x}\right) \sqrt{-1}+\sum_{i=1}^{n} \xi_{i}\left(t,-2 \pi k_{1}^{\dagger} \sqrt{-1}, \ldots,-2 \pi k_{n}^{\dagger} \sqrt{-1}\right)} \\
& \left.+e^{-2 \pi\left(k^{\dagger} \cdot \vec{x}\right) \sqrt{-1}-\sum_{i=1}^{n} \xi_{i}\left(t,-2 \pi k_{1}^{\dagger} \sqrt{-1}, \ldots,-2 \pi k_{n}^{\dagger} \sqrt{-1}\right)}\right), \\
& \psi_{\vec{k}}\left(t, x_{1}, \ldots, x_{n}\right)=\frac{1}{4 \sqrt{-1}}\left(e^{2 \pi(\overrightarrow{(k} \cdot \vec{x}) \sqrt{-1}+\sum_{i=1}^{n} \xi_{i}\left(t, 2 \pi k_{1}^{\dagger} \sqrt{-1}, \ldots, 2 \pi k_{n}^{\dagger} \sqrt{-1}\right)}\right. \\
& +e^{2 \pi\left(k^{\dagger} \cdot \vec{x}\right) \sqrt{-1}-\sum_{i=1}^{n} \xi_{i}\left(t, 2 \pi k_{1}^{\dagger} \sqrt{-1}, \ldots, 2 \pi k_{n}^{\dagger} \sqrt{-1}\right)} \\
& -e^{-2 \pi\left(\vec{k}^{\dagger} \cdot \vec{x}\right) \sqrt{-1}+\sum_{i=1}^{n} \xi_{i}\left(t,-2 \pi k_{1}^{\dagger} \sqrt{-1}, \ldots,-2 \pi k_{n}^{\dagger} \sqrt{-1}\right)} \\
& -e^{-2 \pi(\overrightarrow{(k} \cdot \vec{x}) \sqrt{-1}-\sum_{i=1}^{n} \xi_{i}\left(t,-2 \pi k_{1}^{\dagger} \sqrt{-1}, \ldots,-2 \pi k_{n}^{\dagger} \sqrt{-1}\right)}
\end{aligned}
$$

for $\vec{k} \in \mathbb{Z}^{n}$. Then

$$
\begin{gathered}
\phi_{\vec{k}}\left(0, x_{1}, \ldots, x_{n}\right)=\cos 2 \pi\left(\vec{k}^{\dagger} \cdot \vec{x}\right), \quad \psi_{\vec{k}}\left(0, x_{1}, \ldots, x_{n}\right)=\sin 2 \pi\left(\vec{k}^{\dagger} \cdot \vec{x}\right), \\
\partial_{t}\left(\phi_{\vec{k}}\right)\left(0, x_{1}, \ldots, x_{n}\right)=\partial_{t}\left(\psi_{\vec{k}}\right)\left(0, x_{1}, \ldots, x_{n}\right)=0 .
\end{gathered}
$$

Again we define $\overrightarrow{0} \prec \vec{k}$ if its first nonzero coordinate is a positive integer. By superposition principle and Fourier expansions, we obtain:

Theorem 3.2. The solution of the equation (3.21) subject to (3.22) is

$$
\begin{aligned}
u= & \sum_{\overrightarrow{0} \preceq \vec{k} \in \mathbb{Z}^{n}}\left[b_{0, \vec{k}} \phi_{\vec{k}}\left(t, x_{1}, \ldots, x_{n}\right)+c_{0, \vec{k}} \psi_{\vec{k}}\left(t, x_{1}, \ldots, x_{n}\right)\right. \\
& \left.+b_{1, \vec{k}} \int_{(t)} \phi_{\vec{k}}\left(t, x_{1}, \ldots, x_{n}\right)+c_{1, \vec{k}} \int_{(t)} \psi_{\vec{k}}\left(t, x_{1}, \ldots, x_{n}\right)\right]
\end{aligned}
$$

with

$$
b_{\epsilon, \vec{k}}=\frac{1}{a_{1} a_{2} \cdots a_{n}} \int_{-a_{1}}^{a_{1}} \cdots \int_{-a_{n}}^{a_{n}} g_{\epsilon}\left(x_{1}, \ldots, x_{n}\right) \cos 2 \pi\left(\vec{k}^{\dagger} \cdot \vec{x}\right) d x_{n} \cdots d x_{1}
$$

and

$$
c_{\epsilon, \vec{k}}=\frac{1}{a_{1} a_{2} \cdots a_{n}} \int_{-a_{1}}^{a_{1}} \cdots \int_{-a_{n}}^{a_{n}} g_{\epsilon}\left(x_{1}, \ldots, x_{n}\right) \sin 2 \pi\left(\vec{k}^{\dagger} \cdot \vec{x}\right) d x_{n} \cdots d x_{1} .
$$


The convergence of the series (3.39) is guaranteed by the Kovalevskaya Theorem on the existence and uniqueness of the solution of linear partial differential equations when the functions in (3.22) are analytic.

Example 3.1. Consider the special case

$$
u_{t t}-\left(\partial_{x_{1}}^{2}+x_{1} \partial_{x_{2}}^{2}+x_{2} \partial_{x_{3}}^{2}\right)(u)=0
$$

of (3.21). By (2.71) and (2.72), we have

$$
\begin{gathered}
\xi_{1}\left(t, 2 \pi k_{1}^{\dagger} \sqrt{-1}, 2 \pi k_{1}^{\dagger} 2 \sqrt{-1}, 2 \pi k_{3}^{\dagger} \sqrt{-1}\right) \\
=-4 \pi^{2} t\left[\left(k_{1}^{\dagger}\right)^{2}-\frac{4 \pi^{2} t^{2}}{3}\left(\left(k_{2}^{\dagger}\right)^{4}+2 k_{1}^{\dagger} k_{2}^{\dagger}\left(k_{3}^{\dagger}\right)^{2}\right)+\frac{16 \pi^{4} t^{4}}{3}\left(k_{2}^{\dagger}\right)^{2}\left(k_{3}^{\dagger}\right)^{4}-\frac{64 \pi^{6} t^{6}}{21}\left(k_{3}^{\dagger}\right)^{8}\right] \\
-8 \pi^{3} t^{2}\left[k_{1}^{\dagger}\left(k_{2}^{\dagger}\right)^{2}-\frac{2 \pi^{2} t^{2}}{3}\left(3\left(k_{2}^{\dagger}\right)^{3}\left(k_{3}^{\dagger}\right)^{2}+k_{1}^{\dagger}\left(k_{3}^{\dagger}\right)^{4}\right)+\frac{16 t^{4}}{9} k_{2}^{\dagger}\left(k_{3}^{\dagger}\right)^{6}\right] \sqrt{-1} \\
\xi_{2}\left(t, 2 \pi k_{1}^{\dagger} \sqrt{-1}, 2 \pi k_{1}^{\dagger} 2 \sqrt{-1}, 2 \pi k_{3}^{\dagger} \sqrt{-1}\right) \\
=-4 \pi^{2} t x_{1}\left[\left(k_{2}^{\dagger}\right)^{2}-\frac{4 \pi^{2} t^{2}}{3}\left(k_{3}^{\dagger}\right)^{4}\right]-8 \pi^{3} k_{2}^{\dagger}\left(k_{3}^{\dagger}\right)^{3} t^{2} x_{1} \sqrt{-1}
\end{gathered}
$$

and

$$
\xi_{3}\left(t, 2 \pi k_{1}^{\dagger} \sqrt{-1}, 2 \pi k_{1}^{\dagger} 2 \sqrt{-1}, 2 \pi k_{3}^{\dagger} \sqrt{-1}\right)=-4 \pi^{2}\left(k_{3}^{\dagger}\right)^{2} t x_{2} .
$$

Thus

$$
\begin{aligned}
& \phi_{\vec{k}}\left(t, x_{1}, x_{2}, x_{3}\right)= \\
& \frac{1}{2} \exp \left[4 \pi^{2} t\left[\left(k_{1}^{\dagger}\right)^{2}-\frac{4 \pi^{2} t^{2}}{3}\left(\left(k_{2}^{\dagger}\right)^{4}+2 k_{1}^{\dagger} k_{2}^{\dagger}\left(k_{3}^{\dagger}\right)^{2}\right)+\frac{16 \pi^{4} t^{4}}{3}\left(k_{2}^{\dagger}\right)^{2}\left(k_{3}^{\dagger}\right)^{4}-\frac{64 \pi^{6} t^{6}}{21}\left(k_{3}^{\dagger}\right)^{8}\right]\right. \\
& \left.+4 \pi^{2} t x_{1}\left[\left(k_{2}^{\dagger}\right)^{2}-\frac{4 \pi^{2} t^{2}}{3}\left(k_{3}^{\dagger}\right)^{4}\right]+4 \pi^{2}\left(k_{3}^{\dagger}\right)^{2} t x_{2}\right] \cos \left[2 \pi\left(\vec{k}^{\dagger} \cdot \vec{x}\right)+8 \pi^{3} t^{2}\left[k_{1}^{\dagger}\left(k_{2}^{\dagger}\right)^{2}\right.\right. \\
& \left.\left.-\frac{2 \pi^{2} t^{2}}{3}\left(3\left(k_{2}^{\dagger}\right)^{3}\left(k_{3}^{\dagger}\right)^{2}+k_{1}^{\dagger}\left(k_{3}^{\dagger}\right)^{4}\right)+\frac{16 t^{4}}{9} k_{2}^{\dagger}\left(k_{3}^{\dagger}\right)^{6}\right]+8 \pi^{3} k_{2}^{\dagger}\left(k_{3}^{\dagger}\right)^{3} t^{2} x_{1}\right] \\
& +\frac{1}{2} \exp \left[-4 \pi^{2} t\left[\left(k_{1}^{\dagger}\right)^{2}-\frac{4 \pi^{2} t^{2}}{3}\left(\left(k_{2}^{\dagger}\right)^{4}+2 k_{1}^{\dagger} k_{2}^{\dagger}\left(k_{3}^{\dagger}\right)^{2}\right)+\frac{16 \pi^{4} t^{4}}{3}\left(k_{2}^{\dagger}\right)^{2}\left(k_{3}^{\dagger}\right)^{4}-\frac{64 \pi^{6} t^{6}}{21}\left(k_{3}^{\dagger}\right)^{8}\right]\right. \\
& \left.-4 \pi^{2} t x_{1}\left[\left(k_{2}^{\dagger}\right)^{2}-\frac{4 \pi^{2} t^{2}}{3}\left(k_{3}^{\dagger}\right)^{4}\right]-4 \pi^{2}\left(k_{3}^{\dagger}\right)^{2} t x_{2}\right] \cos \left[2 \pi\left(\vec{k}^{\dagger} \cdot \vec{x}\right)-8 \pi^{3} t^{2}\left[k_{1}^{\dagger}\left(k_{2}^{\dagger}\right)^{2}\right.\right. \\
& \left.\left.-\frac{2 \pi^{2} t^{2}}{3}\left(3\left(k_{2}^{\dagger}\right)^{3}\left(k_{3}^{\dagger}\right)^{2}+k_{1}^{\dagger}\left(k_{3}^{\dagger}\right)^{4}\right)+\frac{16 t^{4}}{9} k_{2}^{\dagger}\left(k_{3}^{\dagger}\right)^{6}\right]-8 \pi^{3} k_{2}^{\dagger}\left(k_{3}^{\dagger}\right)^{3} t^{2} x_{1}\right] \\
& \psi_{\vec{k}}\left(t, x_{1}, x_{2}, x_{3}\right)= \\
& \frac{1}{2} \exp \left[4 \pi^{2} t\left[\left(k_{1}^{\dagger}\right)^{2}-\frac{4 \pi^{2} t^{2}}{3}\left(\left(k_{2}^{\dagger}\right)^{4}+2 k_{1}^{\dagger} k_{2}^{\dagger}\left(k_{3}^{\dagger}\right)^{2}\right)+\frac{16 \pi^{4} t^{4}}{3}\left(k_{2}^{\dagger}\right)^{2}\left(k_{3}^{\dagger}\right)^{4}-\frac{64 \pi^{6} t^{6}}{21}\left(k_{3}^{\dagger}\right)^{8}\right]\right. \\
& \left.+4 \pi^{2} t x_{1}\left[\left(k_{2}^{\dagger}\right)^{2}-\frac{4 \pi^{2} t^{2}}{3}\left(k_{3}^{\dagger}\right)^{4}\right]+4 \pi^{2}\left(k_{3}^{\dagger}\right)^{2} t x_{2}\right] \sin \left[2 \pi\left(\vec{k}^{\dagger} \cdot \vec{x}\right)+8 \pi^{3} t^{2}\left[k_{1}^{\dagger}\left(k_{2}^{\dagger}\right)^{2}\right.\right. \\
& \left.\left.-\frac{2 \pi^{2} t^{2}}{3}\left(3\left(k_{2}^{\dagger}\right)^{3}\left(k_{3}^{\dagger}\right)^{2}+k_{1}^{\dagger}\left(k_{3}^{\dagger}\right)^{4}\right)+\frac{16 t^{4}}{9} k_{2}^{\dagger}\left(k_{3}^{\dagger}\right)^{6}\right]+8 \pi^{3} k_{2}^{\dagger}\left(k_{3}^{\dagger}\right)^{3} t^{2} x_{1}\right] \\
& +\frac{1}{2} \exp \left[-4 \pi^{2} t\left[\left(k_{1}^{\dagger}\right)^{2}-\frac{4 \pi^{2} t^{2}}{3}\left(\left(k_{2}^{\dagger}\right)^{4}+2 k_{1}^{\dagger} k_{2}^{\dagger}\left(k_{3}^{\dagger}\right)^{2}\right)+\frac{16 \pi^{4} t^{4}}{3}\left(k_{2}^{\dagger}\right)^{2}\left(k_{3}^{\dagger}\right)^{4}-\frac{64 \pi^{6} t^{6}}{21}\left(k_{3}^{\dagger}\right)^{8}\right]\right.
\end{aligned}
$$




$$
\begin{aligned}
& \left.-4 \pi^{2} t x_{1}\left[\left(k_{2}^{\dagger}\right)^{2}-\frac{4 \pi^{2} t^{2}}{3}\left(k_{3}^{\dagger}\right)^{4}\right]-4 \pi^{2}\left(k_{3}^{\dagger}\right)^{2} t x_{2}\right] \sin \left[2 \pi\left(\vec{k}^{\dagger} \cdot \vec{x}\right)-8 \pi^{3} t^{2}\left[k_{1}^{\dagger}\left(k_{2}^{\dagger}\right)^{2}\right.\right. \\
& \left.\left.-\frac{2 \pi^{2} t^{2}}{3}\left(3\left(k_{2}^{\dagger}\right)^{3}\left(k_{3}^{\dagger}\right)^{2}+k_{1}^{\dagger}\left(k_{3}^{\dagger}\right)^{4}\right)+\frac{16 t^{4}}{9} k_{2}^{\dagger}\left(k_{3}^{\dagger}\right)^{6}\right]-8 \pi^{3} k_{2}^{\dagger}\left(k_{3}^{\dagger}\right)^{3} t^{2} x_{1}\right]
\end{aligned}
$$

for $\vec{k} \in \mathbb{Z}^{3}$.

\section{Polynomial Representations of Lie Algebras}

In this section, we give three simple examples of applying Lemma 2.1 to polynomial representations of Lie algebras.

Let $\mathbb{F}$ be any field with characteristic 0 and let $n \geq 3$ be an integer. The special orthogonal Lie algebra

$$
s o(n, \mathbb{F})=\sum_{1 \leq i<j \leq n} \mathbb{F}\left(E_{i, j}-E_{j, i}\right),
$$

where $E_{r, s}$ is an $n \times n$ matrix with 1 as its $(r, s)$-entry and 0 as the others. There is a natural representation of $s o(n, \mathbb{F})$ on the algebra $\mathcal{A}=\mathbb{F}\left[x_{1}, \ldots, x_{n}\right]$ of polynomials in $n$ variables:

$$
\left.\left(E_{i, j}-E_{j, i}\right)\right|_{\mathcal{A}}=x_{i} \partial_{x_{j}}-x_{j} \partial_{x_{i}} \quad \text { for } 1 \leq i<j \leq n .
$$

Denote

$$
|\alpha|=\sum_{i=1}^{n} \alpha_{i} \quad \text { for } \alpha=\left(\alpha_{1}, \ldots, \alpha_{n}\right) \in \mathbb{N}^{n}
$$

and

$$
\mathcal{A}_{k}=\sum_{\alpha \in \mathbb{N}^{n},|\alpha|=k} \mathbb{F} x^{\alpha} \quad \text { for } k \in \mathbb{N},
$$

the space of homogeneous polynomials of degree $k$. Set

$$
\mathcal{H}_{k}=\left\{f \in \mathcal{A}_{k} \mid\left(\partial_{x_{1}}^{2}+\cdots+\partial_{x_{n}}^{2}\right)(f)=0\right\}
$$

the space of homogeneous harmonic polynomials of degree $k$. It is well known in harmonic analysis that $\mathcal{H}_{k}$ are irreducible $s o(n, \mathbb{F})$-submodules and

$$
\mathcal{A}_{k}=\mathcal{H}_{k} \oplus\left(x_{1}^{2}+\cdots+x_{n}^{2}\right) \mathcal{A}_{k-2} \quad \text { for } \quad 2 \leq k \in \mathbb{N}
$$

By (2.35), we have:

Theorem 4.1. The following set

$$
\begin{aligned}
& \left\{\sum_{r_{2}, \ldots, r_{k}=0}^{\infty} \frac{(-1)^{r_{2}+\cdots+r_{k}}\left(\begin{array}{c}
r_{2}+\cdots+r_{k} \\
r_{2}, \ldots, r_{n}
\end{array}\right) \prod_{i=2}^{k}\left(\begin{array}{c}
\ell_{i} \\
2 r_{i}
\end{array}\right)}{\left(1+2 \epsilon\left(r_{2}+\cdots+r_{k}\right)\right)\left(\begin{array}{c}
2\left(r_{2}+\cdots+r_{k}\right) \\
2 r_{2}, \ldots, 2 r_{n}
\end{array}\right)} x_{1}^{\epsilon+2\left(r_{2}+\cdots+r_{n}\right)}\right. \\
& \left.\times x_{2}^{\ell_{2}-2 r_{2}} \cdots x_{n}^{\ell_{n}-2 r_{n}} \mid \epsilon \in\{0,1\} ; \ell_{2}, \ldots, \ell_{n} \in \mathbb{N}, \epsilon+\sum_{i=2} \ell_{i}=k\right\}
\end{aligned}
$$

forms a basis of $\mathcal{H}_{k}$. 
Recall the special linear Lie algebra

$$
s l(n, \mathbb{F})=\sum_{i \neq j} \mathbb{F} E_{i, j}+\sum_{i=1}^{n-1} \mathbb{F}\left(E_{i, i}-E_{i+1, i+1}\right) .
$$

Note

$$
H=\sum_{i=1}^{n-1} \mathbb{F}\left(E_{i, i}-E_{i+1, i+1}\right)
$$

is a Cartan subalgebra of $\operatorname{sl}(n, \mathbb{F})$. Take $\left\{E_{i, j} \mid 1 \leq i<j \leq n\right\}$ as positive root vectors. Let

$$
\mathcal{Q}=\mathbb{F}\left(x_{1}, \ldots, x_{n}, y_{1}, \ldots, y_{n}\right),
$$

the space of rational functions in $x_{1}, \ldots, x_{n}, y_{1}, \ldots, y_{n}$. Define a representation of $s l(n, \mathbb{F})$ on $\mathcal{Q}$ via

$$
\left.E_{i, j}\right|_{\mathcal{Q}}=x_{i} \partial_{x_{j}}-y_{j} \partial_{y_{i}} \quad \text { for } i, j \in \overline{1, n} .
$$

A nonzero function $f \in \mathcal{Q}$ is called singular if

$$
E_{i, j}(f)=0 \quad \text { for } 1 \leq i<j \leq n
$$

and there exist a linear function $\lambda$ on $H$ such that

$$
h(f)=\lambda(h) f \quad \text { for } h \in H .
$$

Set

$$
\zeta=\sum_{i=1}^{n} x_{i} y_{i}
$$

Then

$$
\xi(\zeta)=0 \quad \text { for } \xi \in \operatorname{sl}(n, \mathbb{F}) .
$$

Lemma 4.2. Any singular function in $\mathcal{Q}$ is a rational function in $x_{1}, y_{n}, \zeta$.

Proof. Let $f \in \mathcal{Q}$ be a singular function. We can write

$$
f=g\left(x_{1}, \ldots, x_{n-1}, \zeta, y_{1}, \ldots, y_{n}\right)
$$

as a rational functions in $x_{1}, \ldots, x_{n-1}, \zeta, y_{1}, \ldots, y_{n}$. By (4.11), (4.12) and (4.15), we have:

$$
E_{i, n}(f)=-y_{n} \partial_{y_{i}}(g)=0 \quad \text { for } i \in \overline{1, n-1},
$$

equivalently,

$$
\partial_{y_{i}}(g)=0 \quad \text { for } i \in \overline{1, n-1} .
$$

Thus (4.11) and (4.19) imply

$$
E_{1, i}(g)=x_{1} \partial_{x_{i}}(g)=0 \quad \text { for } i \in \overline{2, n-1},
$$


that is,

$$
\partial_{x_{i}}(g)=0 \quad \text { for } i \in \overline{2, n-1},
$$

Therefore, $g$ is independent of $x_{2}, \ldots, x_{n-1}$ and $y_{1}, \ldots, y_{n-1}$.

Set

$$
\mathcal{A}_{\ell_{1}, \ell_{2}}=\sum_{\alpha, \beta \in \mathbb{N}^{n} ;|\alpha|=\ell_{1},|\beta|=\ell_{2}} \mathbb{F} x^{\alpha} y^{\beta} \quad \text { for } \ell_{1}, \ell_{2} \in \mathbb{N} .
$$

Then $\mathcal{A}_{\ell_{1}, \ell_{2}}$ is a finite-dimensional $\operatorname{sl}(n, \mathbb{F})$-submodule by (4.11). Recall that the fundamental weights $\lambda_{1}, . ., \lambda_{n-1}$ are linear functions on $H$ such that

$$
\lambda_{i}\left(E_{j, j}-E_{j+1, j+1}\right)=\delta_{i, j} \quad \text { for } i, j \in \overline{1, n-1} .
$$

The function $x_{1}^{\ell_{1}} y_{n}^{\ell_{2}}$ is a singular function of weight $\ell_{1} \lambda_{1}+\ell_{2} \lambda_{n-1}$. According to the above lemma, any singular polynomial in $\mathcal{A}_{\ell_{1}, \ell_{2}}$ must be of the form $a x_{1}^{\ell_{1}-i} y_{n}^{\ell_{2}-i} \zeta^{i}$ for some $0 \neq a \in \mathbb{F}$ and $i \in \mathbb{N}$. Define

$$
V_{\ell_{1}, \ell_{2}}=\text { the submodule generated by } x_{1}^{\ell_{1}} y_{n}^{\ell_{2}} \text {. }
$$

According to Weyl's Theorem of completely reducibility, $\mathcal{A}_{\ell_{1}, \ell_{2}}$ is a direct sum of its irreducible submodules, which are generated by its singular polynomials. So $V_{\ell_{1}, \ell_{2}}$ is an irreducible highest weight module with the highest weight $\ell_{1} \lambda_{1}+\ell_{2} \lambda_{n-1}$ and

$$
\mathcal{A}_{\ell_{1}, \ell_{2}}=V_{\ell_{1}, \ell_{2}} \oplus \zeta \mathcal{A}_{\ell_{1}-1, \ell_{2}-1}
$$

as a direct sum of two $\operatorname{sl}(n, \mathbb{F})$-submodules, where we treat $V_{i, j}=\{0\}$ if $\{i, j\} \not \subset \mathbb{N}$.

Denote

$$
\Delta=\sum_{i=1}^{n} \partial_{x_{i}} \partial_{y_{i}}
$$

It can be verified that

$$
\xi \Delta=\Delta \xi \quad \text { for } \xi \in \operatorname{sl}(n, \mathbb{F}),
$$

as operators on $\mathcal{Q}$. Set

$$
\mathcal{H}_{\ell_{1}, \ell_{2}}=\left\{f \in \mathcal{A}_{\ell_{1}, \ell_{2}} \mid \Delta(f)=0\right\}
$$

Since $\Delta\left(x_{1}^{\ell_{1}} y_{n}^{\ell_{2}}\right)=0$, we have

$$
V_{\ell_{1}, \ell_{2}} \subset \mathcal{H}_{\ell_{1}, \ell_{2}}
$$

by (4.24) and (4.27). On the other hand,

$$
\mathcal{A}_{\ell_{1}, \ell_{2}}=\bigoplus_{i=0}^{\infty} \zeta^{i} V_{\ell_{1}-i, \ell_{2}-i}
$$

by (4.25) and induction. Note

$$
\Delta \zeta=n+\zeta \Delta+\sum_{i=1}^{n}\left(x_{i} \partial_{x_{i}}+y_{i} \partial_{y_{i}}\right)
$$


as operators on $\mathcal{Q}$. Thus

$$
\Delta\left(\zeta^{i} g\right)=\sum_{r=1}^{i}\left(n+\ell_{1}+\ell_{2}-2 r\right)\left(\zeta^{i-1} g\right)=i\left(n+\ell_{1}+\ell_{2}-i-1\right) \zeta^{i-1} g
$$

for $i \in \mathbb{N}+1, g \in V_{\ell_{1}-i, \ell_{2}-i}$. Hence

$$
\mathcal{H}_{\ell_{1}, \ell_{2}} \bigcap \zeta \mathcal{A}_{\ell_{1}-1, \ell_{2}-1}=\{0\} .
$$

Therefore,

$$
V_{\ell_{1}, \ell_{2}}=\mathcal{H}_{\ell_{1}, \ell_{2}}
$$

by (4.25) and (4.29). Now Lemma 2.1 gives:

Theorem 4.3. The set

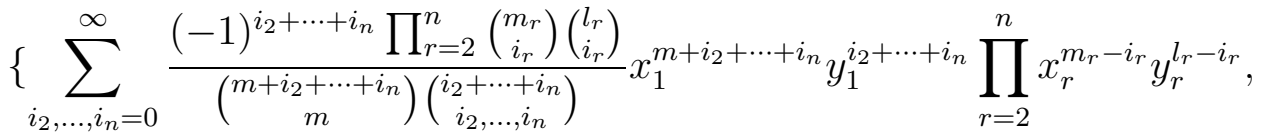

$$
\begin{aligned}
& \sum_{i_{2}, \ldots, i_{n}=0}^{\infty} \frac{(-1)^{i_{2}+\cdots+i_{n}} \prod_{r=2}^{n}\left(\begin{array}{c}
m_{r}^{\prime} \\
i_{r}
\end{array}\right)\left(\begin{array}{c}
l_{r} \\
i_{r}
\end{array}\right)}{\left(\begin{array}{c}
m^{\prime}+i_{2}+\cdots+i_{n} \\
m^{\prime}
\end{array}\right)\left(\begin{array}{c}
i_{2}+\cdots+i_{n} \\
i_{2}, \ldots, i_{n}
\end{array}\right)} x_{1}^{i_{2}+\cdots+i_{n}} y_{1}^{m^{\prime}+i_{2}+\cdots+i_{n}} \prod_{r=2}^{n} x_{r}^{m_{r}^{\prime}-i_{r}} y_{r}^{l_{r}^{\prime}-i_{r}} \\
& \left.\mid m, m^{\prime}, m_{r}, n_{r} \in \mathbb{N} ; m+\sum_{r=2}^{n} m_{r}=\sum_{r=2}^{n} m_{r}^{\prime}=\ell_{1}, \sum_{r=2}^{n} l_{r}=m^{\prime}+\sum_{r=2}^{n} l_{r}^{\prime}=\ell_{2}\right\}
\end{aligned}
$$

for a basis of $V_{\ell_{1}, \ell_{2}}$.

In the Lie algebra $s l(7, \mathbb{F})$, we set

$$
\begin{gathered}
h_{1}=-2 E_{2,2}+E_{3,3}+E_{4,4}+2 E_{5,5}-E_{6,6}-E_{7,7}, \quad h_{2}=E_{2,2}-E_{3,3}-E_{5,5}+E_{6,6}, \\
E_{1}=\sqrt{2}\left(E_{1,2}-E_{5,1}\right)-E_{3,7}+E_{4,6}, \quad E_{2}=E_{2,3}-E_{6.5}, \\
E_{3}=\left[E_{1}, E_{2}\right]=\sqrt{2}\left(E_{1,3}-E_{6,1}\right)+E_{2,7}-E_{4,5}, \\
E_{4}=\left[E_{1}, E_{3}\right] / 2=\sqrt{2}\left(E_{1,7}-E_{4,1}\right)+E_{6,2}-E_{5,3}, \\
E_{5}=\left[E_{1}, E_{4}\right] / 3=E_{4,2}-E_{5,7}, \quad E_{6}=\left[E_{5}, E_{2}\right]=E_{4,3}-E_{6,7}, \\
F_{1}=\sqrt{2}\left(E_{2,1}-E_{1,5}\right)-E_{7,3}+E_{6,4}, \quad F_{2}=E_{3,2}-E_{5,6}, \\
F_{3}=\sqrt{2}\left(E_{3,1}-E_{1,6}\right)+E_{7,2}-E_{5,4}, \quad F_{5}=E_{2,4}-E_{7,5}, \\
F_{4}=\sqrt{2}\left(E_{7,1}-E_{1,4}\right)+E_{2,6}-E_{3,5}, \quad F_{6}=E_{3,4}-E_{7,6} .
\end{gathered}
$$

Then the exceptional Lie algebra of type $G_{2}$ is the Lie subalgebra

$$
\mathcal{G}_{2}=\mathbb{C} h_{1}+\mathbb{C} h_{2}+\sum_{i=1}^{6}\left(\mathbb{C} E_{i}+\mathbb{C} F_{i}\right)
$$

of $\operatorname{sl}(7, \mathbb{F})(\mathrm{cf} .[\mathrm{H}])$. Its Cartan subalgebra

$$
H=\mathbb{C} h_{1}+\mathbb{C} h_{2} .
$$


We choose $\left\{E_{1}, E_{2}, \ldots, E_{6}\right\}$ as positive root vectors.

Let $Q$ be the space of rational functions in $\left\{x_{i} \mid i \in \overline{1,7}\right\}$ and define a representation of $\mathcal{G}_{2}$ on $Q$ via

$$
\left.E_{i, j}\right|_{Q}=x_{i} \partial_{x_{j}} \quad \text { for } i, j \in \overline{1,7} .
$$

A nonzero function $f \in Q$ is called singular with respect to $\mathcal{G}_{2}$ if

$$
E_{i}(f)=0 \quad \text { for } i \in \overline{1,6}
$$

and (4.13) holds. Define

$$
\eta=x_{1}^{2}+2 x_{2} x_{5}+2 x_{3} x_{6}+2 x_{4} x_{7} .
$$

It can be verified that

$$
\xi(\eta)=0 \quad \text { for } \xi \in \mathcal{G}_{2} .
$$

Lemma 4.4. Any singular function in $Q$ with respect to $\mathcal{G}_{2}$ must be a rational function in $x_{4}$ and $\eta$.

Proof. Let $f$ a singular function in $Q$. We can write

$$
f=\varphi\left(x_{1}, \ldots, x_{6}, \eta\right)
$$

as a rational function in $x_{1}, \ldots, x_{6}, \eta$. Note

$$
E_{5}(f)=\left(x_{4} \partial_{x_{2}}-x_{5} \partial_{x_{7}}\right)\left(\varphi\left(x_{1}, \ldots, x_{6}, \eta\right)\right)=x_{4} \partial_{x_{2}}(\varphi)=0
$$

and

$$
E_{6}(f)=\left(x_{4} \partial_{x_{3}}-x_{6} \partial_{x_{7}}\right)\left(\varphi\left(x_{1}, \ldots, x_{6}, \eta\right)\right)=x_{4} \partial_{x_{3}}(\varphi)=0
$$

So

$$
\partial_{x_{2}}(\varphi)=\partial_{x_{3}}(\varphi)=0 \text {. }
$$

Next

$$
E_{2}(f)=\left(x_{2} \partial_{x_{3}}-x_{6} \partial_{x_{5}}\right)\left(\varphi\left(x_{1}, \ldots, x_{6}, \eta\right)\right)=-x_{6} \partial_{x_{5}}(\varphi)=0,
$$

that is,

$$
\partial_{x_{5}}(\varphi)=0
$$

Moreover,

$$
E_{3}(f)=\left(\sqrt{2}\left(x_{1} \partial_{x_{3}}-x_{6} \partial_{x_{1}}\right)+x_{2} \partial_{x_{7}}-x_{4} \partial_{x_{5}}\right)\left(\varphi\left(x_{1}, \ldots, x_{6}, \eta\right)\right)=-\sqrt{2} x_{6} \partial_{x_{1}}(\varphi)=0,
$$

which implies

$$
\partial_{x_{1}}(\varphi)=0
$$

Furthermore,

$$
E_{2}(f)=\left(\sqrt{2}\left(x_{1} \partial_{x_{2}}-x_{5} \partial_{x_{1}}\right)-x_{3} \partial_{x_{7}}+x_{4} \partial_{x_{6}}\right)\left(\varphi\left(x_{1}, \ldots, x_{6}, \eta\right)\right)=x_{4} \partial_{x_{6}}(\varphi)=0
$$


yields

$$
\partial_{x_{6}}(\varphi)=0 .
$$

According to (4.53), (4.55), (4.57) and (4.59), $\varphi$ is independent of $x_{1}, x_{2}, x_{3}, x_{5}, x_{6}$.

Set

$$
\Delta^{\prime}=\partial_{x_{2}}^{2}+2 \partial_{x_{2}} \partial_{x_{5}}+2 \partial_{x_{3}} \partial_{x_{6}}+2 \partial_{x_{4}} \partial_{x_{7}} .
$$

It can be verified that

$$
\Delta^{\prime} \xi=\xi \Delta^{\prime} \quad \text { for } \xi \in \mathcal{G}_{2}
$$

and

$$
\Delta^{\prime} \eta=\eta \Delta^{\prime}+14+4 \sum_{i=1}^{7} x_{i} \partial_{x_{i}}
$$

as operators on $Q$. The fundamental weight $\lambda_{1}$ is a linear function on $H$ such that

$$
\lambda\left(h_{1}\right)=1, \quad \lambda_{1}\left(h_{2}\right)=0 .
$$

For $k \in \mathbb{N}, x_{4}^{k}$ is a singular function with weight $k \lambda_{1}$. Note that

$$
\mathcal{B}_{k}=\sum_{\alpha \in \mathbb{N}^{7} ;|\alpha|=k} \mathbb{F} x^{\alpha}
$$

is a finite-dimensional $\mathcal{G}_{2}$-submodule. Let

$$
V_{k}=\text { the submodule generated by } x_{4}^{k} \text {. }
$$

Then $V_{k}$ is an irreducible highest weight submodule of $\mathcal{B}_{k}$ with highest weight $k \lambda_{1}$. By similar arguments as those in the above of Theorem 4.3, we can prove

$$
V_{k}=\left\{f \in \mathcal{B}_{k} \mid \Delta^{\prime}(f)=0\right\} .
$$

Theorem 4.5. The set

$$
\begin{aligned}
& \left\{\sum_{i_{2}, i_{3}, i_{4}=0}^{\infty} \frac{(-1)^{i_{2}+i_{3}+i_{4}} \prod_{r=2}^{3}\left(\begin{array}{c}
m_{r} \\
i_{r}
\end{array}\right)\left(\begin{array}{c}
m_{i_{r}+r} \\
i_{r}
\end{array}\right)}{\left(1+2 \epsilon\left(i_{2}+i_{3}+i_{4}\right)\right)\left(\begin{array}{c}
2\left(i_{2}+i_{2}+i_{4}\right) \\
i_{2}, i_{4}, i_{2}+i_{3}+i_{4}
\end{array}\right)} x_{1}^{\epsilon+2\left(i_{2}+i_{3}+i_{4}\right)} \prod_{s=2}^{4} x_{s}^{m_{s}-i_{s}} x_{4+s}^{m_{4+s}-i_{s}}\right. \\
& \left.\mid \epsilon \in\{0,1\}, m_{2}, \ldots, m_{7} \in \mathbb{N} ; \epsilon+\sum_{r=2}^{7} m_{r}=k\right\}
\end{aligned}
$$

forms a basis of $V_{k}$.

\section{References}


[A] A. V. Aksenov, Symmetries and fundamental solutions of multidimensional generalized axi-symmetric equations Laplace Equation, Differentsial'nye Uravneniya 29 (1993), 11-

[BG1] J. Barros-Neto and I. M. Gel'fand, Fundamental solutions for the Tricomi operator, Duke Math. J. 98 (1999), 465-483.

[BG2] J. Barros-Neto and I. M. Gel'fand, Fundamental solutions for the Tricomi operator II, Duke Math. J. 111 (2002), 561-584.

[B] Yu. Yu. Berest, Weak invariants of local transformation groups, Differentsial'nye Uravneniya 29 (1993), 1796.

[I1] N. H. Ibragimov, Transformation groups applied to mathematical physics, Nauka, 1983.

[I2] N. H. Ibragimov, Lie Group Analysis of Differential Equations, Volume 2, CRC Handbook, CRC Press, 1995.

[H] J. E. Humphreys, Introduction to Lie Algebras and Representation Theory, SpringerVerlag New York Inc., 1972.

[X1] X. Xu, Differential invariants of classical groups, Duke Math. J. 94 (1998), 543-572.

[X2] X. Xu, Tree diagram Lie algebras of differential operators and evolution partial differential equations, J. Lie Theory 16 (2006), no. 4, 691-718. 